\title{
Fuzzy Group Decision Making with Incomplete Information Guided by Social Influence
}

\author{
Nicola Capuano, Francisco Chiclana, Hamido Fujita, Enrique Herrera-Viedma, Vincenzo Loia
}

\begin{abstract}
A promising research area in the field of Group Decision Making (GDM) is the study of interpersonal influence and its impact on the evolution of experts' opinions. In conventional GDM models, a group of experts express their individual preferences on a finite set of alternatives, then preferences are aggregated and the best alternative, satisfying the majority of experts, is selected. Nevertheless, in real situations, experts form their opinions in a complex interpersonal environment where preferences are liable to change due to social influence. In order to take into account the effects of social influence during the GDM process, we propose a new influence-guided GDM model based on the following assumptions: experts influence each other and the more an expert trusts in another expert, the more his opinion is influenced by that expert. The effects of social influence are especially relevant to cases when, due to domain complexity, limited expertise or pressure to make a decision, an expert is unable to express preferences on some alternatives, i.e. in presence of incomplete information. The proposed model adopts fuzzy rankings to collect both experts' preferences on available alternatives and trust statements on other experts. Starting from collected information, possibly incomplete, the configuration and the strengths of interpersonal influences are evaluated and represented through a Social Influence Network (SIN). The SIN, in its turn, is used to estimate missing preferences and evolve them by simulating the effects of experts' interpersonal influence before aggregating them for the selection of the best alternative. The proposed model has been experimented with synthetic data to demonstrate the influence driven evolution of opinions and its convergence properties.
\end{abstract}

Index Terms - Fuzzy Preference Relation, Group Decision Making, Social Influence

\section{INTRODUCTION}

GDM is a process by which a group of experts collectively makes a choice among a finite set of available alternatives. GDM has been widely studied since it has applications in

This work was supported by the FEDER funds under Grant TIN201675850-R.

N. Capuano is with the Department of Information, Electric Engineering and Applied Mathematics, University of Salerno, Via Giovanni Paolo II 132, 84084 Fisciano (SA), Italy. (e-mail: ncapuano@unisa.it).

F. Chiclana is with the School of Computer Science and Informatics, Faculty of Technology, De Montfort University, Leicester LE1 9BH, UK (e-mail: chiclana@dmu.ac.uk).

E. Herrera-Viedma is with the Department of Computer Science and Artificial Intelligence, University of Granada, Granada 18071, Spain (e-mail: viedma@decsai.ugr.es).

H. Fujita is with the Faculty of Software and Information Science, Iwate Prefectural University, Iwate, Japan (e-mail: HFujita-799@acm.org)

V. Loia is with the Department of Management and Innovation Systems, University of Salerno, Via Giovanni Paolo II 132, 84084 Fisciano (SA), Italy (e-mail: loia@unisa.it) many fields. For this reason, many different approaches have been proposed so far for the representation of experts' opinion, for their aggregation, for the selection of the best alternative and for consensus reaching [1]-[5]. In a GDM process, experts are usually let free to interact and discuss each other exchanging opinions. During these interactions, experts with a wider background, experience and knowledge are capable of influencing other experts. So, after a discussion, the preferences of each expert may undergo a modification due to social influence. Influence modelling and the appraisal of its effect on opinion change has been studied in [6] and [7]. Influence is capable of playing a key role in decision making; despite that, the introduction of GDM models that takes into account social influence have just recently been proposed in [8] and [9].

Following these works, this paper presents an alternative influence-guided GDM model that, instead of considering predefined levels of social influence, models influence based on available information regarding experts' interpersonal trust. Elaborating on the definitions provided in [10], the concept of trust is interpreted in the proposed framework as the belief of an expert in the capability of another expert in finding the correct solution to a specific problem.

Interpersonal trust has been already used to improve the outcomes of a GDM process. According to the models proposed in [11] and [12], each expert is explicitly asked to express their fuzzy trust statements on the other experts. Such statements are then aggregated and a global level of trust is calculated, associated to each expert and used to weight their opinions in the aggregation step. Instead, we propose to use trust statements to let the opinions of each expert evolve by incorporating elements captured from the opinion expressed by other experts he trusts.

Especially in the case of uncertainty it is generally believed that people are influenced by the opinions of people they trust. Information regarding the level of interpersonal trust among the experts is then collected together with opinions on problem alternatives (that may be incomplete). According to [9], incomplete opinions are then completed with data injected from trusted experts. In addition with respect to [9], such opinions are further modified by simulating their evolution due to social influence. It is also demonstrated that, under certain assumptions, the evolution of opinions due to influence converges to a final collective opinion. If assumptions are not met, standard aggregation approaches are used to select the best alternative. The paper is organized as follows: section II introduces some background concepts on GDM and related topics while section III discusses the proposed methodology. Section IV presents 
an in silico simulation of the proposed methodology that illustrates its operational steps and properties as well as and its advantages and peculiarities with respect to alternative methods. Conclusions are pointed out in section V.

\section{PRELIMINARIES ON GROUP DECISION MAKING}

A GDM problem is characterized by a group of experts $E=\left\{e_{1}, \ldots, e_{m}\right\}$, each with their own knowledge, ideas, experience and motivation, who express their preferences on a finite set of alternatives $X=\left\{x_{1}, \ldots, x_{n}\right\}$ to achieve a common solution. Expert preferences can be expressed in form of rankings [13] (alternatives are ordered from the best to the worst), utility vectors [14] (a utility value is assigned to each alternative), fuzzy estimates [15] (a linguistic evaluation, translated into a fuzzy number, is assigned to each alternative), preference relations [16] (for every pair of alternatives, a preferred one is selected) or fuzzy preference relations [17][18] (a degree of preference for each alternative over any other is assigned).

Among the existing models, Fuzzy Preference Relations (FPRs) are one of the most diffused. They ensure a high degree of expressiveness in the definition of preferences and, at the same time, translation techniques are available to convert preference information from every other representation model [19][20]. A FPR $P$ on a set of alternatives $X$ can be formally defined as a fuzzy set on $X \times X$ with a membership function $\mu_{P}: X \times X \rightarrow[0,1]$ so that $[21]:$

$$
\mu_{P}\left(x_{i}, x_{j}\right)= \begin{cases}1 & \text { if } x_{i} \text { is definitely preferred to } x_{j}, \\ x \in(0.5,1) & \text { if } x_{i} \text { is slightly preferred to } x_{j}, \\ 0.5 & \text { if } x_{i} \text { and } x_{j} \text { are evenly preferred, } \\ y \in(0,0.5) & \text { if } x_{j} \text { is slightly preferred to } x_{i} \\ 0 & \text { if } x_{j} \text { is definitely preferred to } x_{i} .\end{cases}
$$

A FPR $P$ can be represented as a $n \times n$ matrix $P=\left(p_{i j}\right)$ where $p_{i j}=\mu_{P}\left(x_{i}, x_{j}\right)$. A FPR satisfying the additive reciprocity property so that $p_{i j}+p_{j i}=1 \forall i, j \in\{1, \ldots, n\}$, is said to be reciprocal. This means that the preference relation is asymmetric, i.e. if $x_{i}$ is preferred to $x_{j}$ then $x_{j}$ is not preferred to $x_{i}$ and, as a consequence, $p_{i i}=0.5 \forall i \in\{1, \ldots, n\}$ (i.e. any alternative is never preferred to itself). According to [22], a FPR satisfying the additive transitivity property, $p_{i j}+p_{j k}+p_{k i}=$ $1.5 \forall i, j, k \in\{1, \ldots, n\}$, is said to be additive consistent. Additive transitivity is frequently used for repairing inconsistencies or to estimate missing preferences.

Once experts have expressed their preferences, $m$ individual FPRs $P_{1}, \ldots, P_{m}$ are available where $P_{k}=\left(p_{k i j}\right)$ for $k \in\{1, \ldots, m\}$ and $i, j \in\{1, \ldots, n\}$. Several aggregation rules have been proposed so far to obtain a collective FPR $P$ from a set of individual ones. Among them, the Ordered Weighted Average (OWA) family of operators [23][24] is often adopted. A OWA operator of dimension $m$ is a function OWA: $[0,1]^{m} \rightarrow[0,1]$ associated with a list of weights $W=\left(w_{1}, \ldots, w_{m}\right) \in[0,1]^{m}$ such that $\sum_{k=1}^{m} w_{k}=1$. Let $\left(p_{1}, \ldots, p_{m}\right)$ be a list of preference values to aggregate, the OWA operator is defined as:
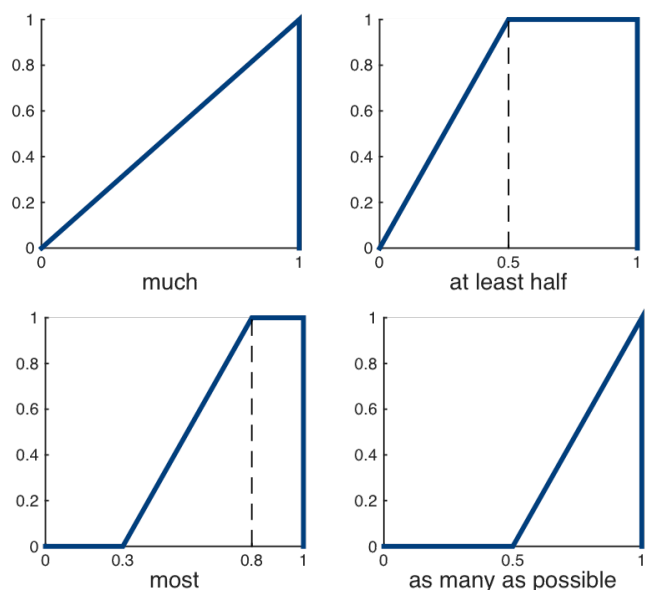

Fig. 1. Example of proportional fuzzy quantifiers

$$
O W A\left(p_{1}, \ldots, p_{m}\right)=\sum_{k=1}^{m} w_{k} p_{\sigma(k)}
$$

where $\sigma:\{1, \ldots m\} \rightarrow\{1, \ldots m\}$ is a permutation function such that $p_{\sigma(k)} \geq p_{\sigma(k+1)}$ for each $k \in\{1, \ldots, m-1\}$. Thus, the OWA collective preference $p_{i j}$ is obtained as: $p_{i j}=$ $O W A\left(p_{1 i j}, \ldots, p_{m i j}\right)$ for $i, j \in\{1, \ldots, n\}$.

The behavior of an OWA operator strictly depends on the used weight vector. In [26], the authors proposed to initialize the weight vector starting from a non-decreasing proportional fuzzy quantifier to let OWA assumes the behavior of soft majority. In this way it is possible to obtain a collective evaluation in which the opinions of most of the experts involved in the decision problem are considered.

A proportional fuzzy quantifier $Q$ is as fuzzy subset of the unit interval $[0,1]$ where, for any $y \in[0,1], \mu_{Q}(y)$ represents the degree to which the proportion $y$ is compatible with the meaning of the quantifier $Q$ [27][28]. A non-decreasing proportional fuzzy quantifier satisfies the additional property: $\mu_{Q}\left(y_{1}\right) \geq$ $\mu_{Q}\left(y_{2}\right)$ when $y_{1}>y_{2}$. Figure 1 shows several examples of non-decreasing proportional fuzzy quantifiers with membership functions:

$$
\mu_{Q}(y)= \begin{cases}0 & \text { if } y<a \\ (y-a) /(b-a) & \text { if } a \leq y \leq b, \\ 1 & \text { if } y>b .\end{cases}
$$

with $a, b, y \in[0,1]$. For example, the parameters $(a, b)$ of the quantifiers shown in Figure 1 are the following: $(0,1),(0$, $0.5),(0.3,0.8)$ and $(0.5,1)$ respectively. The quantifier to be applied should be selected to reflect the fusion strategy that the decision makers would apply. Starting from the selected quantifier, the weights of an OWA operator of dimension $m$ are computed as follows [23]:

$$
w_{k}=\mu_{Q}\left(\frac{k}{m}\right)-\mu_{Q}\left(\frac{k-1}{m}\right) ; k \in\{1, \ldots, m\} .
$$

After having selected a non-decreasing proportional fuzzy quantifier $Q$, extending the notation to matrices, we can write $P=O W A_{Q}\left(P_{1}, \ldots, P_{m}\right)$ where $O W A_{Q}$ is the OWA operator 
initialised with the weights coming from the quantifier $Q$. Alternative aggregation rules, based on OWA and implementing the concept of soft majority have been described in [25].

Once the individual FPRs have been aggregated, the available alternatives must be ranked from best to worst by associating a score value $\phi\left(x_{i}\right)$ to any $x_{i} \in X$. In [26] the Quantifier Guided Dominance Degree (QGDD) was defined to calculate the dominance that one alternative has over all the others in a fuzzy majority sense:

$$
\phi\left(x_{i}\right)=O W A_{Q}\left(p_{i j} ; j=1, \ldots, n ; j \neq i\right) .
$$

In the same paper a Quantifier Guided Non-Dominance Degree was defined to calculate the degree which a given alternative is not dominated by a fuzzy majority of the remaining ones. Instead, in [21] the score value of each alternative $x_{i}$ is calculated in terms of Net Flow i.e. the difference between the degree of preference of $x_{i}$ over all the other alternatives and the degree of preference of all the other alternatives over $x_{i}$.

\section{A. Dealing with Incomplete Information}

Sometimes, due to domain complexity, limited expertise or pressure to make a decision, it may be difficult or even impossible for an expert to express a preference on every pair of alternatives. This results in incomplete FPRs where missing values have to be estimated. Several methods have been proposed so far for this purpose, like the ones in [20][29]-[35]. In particular, we focus on the model discussed in [30] because missing values are estimated combining additive reciprocity and additive transitivity properties.

When an FPR $P$ is additive consistent, an unknown element can be obtained combining known elements of $P$. Even when user defined FPRs are partially consistent, additive transitivity can be used to identify missing values that are as consistent as possible with the defined ones through a set of estimators. Given an unknown value $p_{i j}$, the following partial consistency based estimated values can be computed using alternative $k$ :

$\varepsilon_{k 1}\left(p_{i j}\right)=p_{i k}+p_{k j}-0.5 \forall k: p_{i k}$ and $p_{k j}$ are defined;

$\varepsilon_{k 2}\left(p_{i j}\right)=p_{k j}-p_{k i}+0.5 \forall k: p_{k i}$ and $p_{k j}$ are defined;

$\varepsilon_{k 3}\left(p_{i j}\right)=p_{i k}-p_{j k}+0.5 \forall k: p_{i k}$ and $p_{j k}$ are defined.

A missing value $p_{i j}$ can be so estimated by averaging all partial consistency based estimated values as follows:

$$
\varepsilon\left(p_{i j}\right)=\frac{\sum_{k=1 ; k \neq i ; k \neq j}^{n}\left(\varepsilon_{k 1}\left(p_{i j}\right)+\varepsilon_{k 2}\left(p_{i j}\right)+\varepsilon_{k 3}\left(p_{i j}\right)\right)}{3(n-2)} .
$$

The generation of missing values is done in several iterations. In each iteration new values are generated based on those previously known. When new values cannot be generated, the process stops.

The method uses the preferences expressed on a given alternative to infer missing preferences for the same alternative. If no preferences are available for a given alternative, no estimated value can be generated for it. This happens when, to estimate some $p_{i j}$ for $i, j \in\{1, \ldots, n\}$, both $p_{i k}$ and $p_{k j}$ are undefined for all $k$. In [36], the authors refer to this case as an ignorance situation and suggest to use seed values to initialize missing preferences and, then, to apply an iterative process based on (6)-(7) to obtain the final estimates. Four different ways to obtain seed values are proposed:

- indifference: undefined preferences are initially set to 0.5 ;

- alternative proximity: seed values are obtained from the preference values given by the same expert to similar alternatives;

- collective seed value: seed values are chosen from the collective FPR computed by aggregating partial individual FPRs provided by the experts;

- expert proximity: seed values are chosen from the FPRs provided by the experts nearest to the expert whose FPR has to be completed, distances between experts are computed by averaging the absolute differences between defined preferences.

The first approach is useful when there are no external sources of information about the problem and when a high FPRs consistency level is required. The second approach implies having additional information on alternatives allowing to define a distance measure between them. The third and fourth approaches, making the opinions of the experts closer, are useful when a fast consensus is needed. In addition, the fourth approach is also able to maintain high the FPRs consistency level.

\section{B. Experts' Importance, Consistency and Trust}

When individual FPRs are aggregated into the collective one, it is possible to weight the contribution of each expert in order to reflect their different backgrounds and levels of knowledge about the problem. This is usually achieved by associating an importance degree $u_{k} \in[0,1]$ to each expert $e_{k} \in E$ and implementing a specific aggregation operator, that appropriately takes into account such importance degrees in deriving the collective FPR [37]-[39]. In [37] the Induced OWA (IOWA) operator is introduced for this purpose. With IOWA, the reordering of the set of values to aggregate is induced by the reordering of a set of values associated with them. Based on the same principle, the Importance IOWA operator (I-IOWA) was defined in [38] to consider the importance of each preference (i.e. that of the expert expressing such preference) during the aggregation step. This is elaborated below.

Let $U=\left(u_{1}, \ldots, u_{m}\right)$ be the importance degree of each expert of $E,\left(p_{1}, \ldots, p_{m}\right)$ the list of preference values to aggregate (where $p_{k}$ is expressed by expert $e_{k}$ ) and $Q$ a non-decreasing proportional fuzzy quantifier, the I-IOWA operator is defined as follows:

$$
I-I O W A_{Q}\left(\left(p_{1}, u_{1}\right), \ldots,\left(p_{m}, u_{m}\right)\right)=\sum_{k=1}^{m} w_{k} p_{\sigma(k)}
$$

where $\sigma:\{1, \ldots m\} \rightarrow\{1, \ldots m\}$ is a permutation function such that $u_{\sigma(k)} \geq u_{\sigma(k+1)}$ for each $k \in\{1, \ldots, m-1\}$ and the $k$-th weight $w_{k}$ is obtained as follows:

$$
w_{k}=\mu_{Q}\left(\frac{S(k)}{S(m)}\right)-\mu_{Q}\left(\frac{S(k-1)}{S(m)}\right) ; k \in\{1, \ldots, m\} .
$$

where $S(k)=\sum_{l=1}^{k} u_{\sigma(k)}$. Extending the notation to matrices, 
given a set of individual FPRs $P_{1}, \ldots, P_{m}$ and a vector of experts' importance degrees $U$, the collective FPR $P$ that takes into account the importance of each expert can be obtained as $P=I-O W A_{Q}\left(\left(P_{1}, u_{1}\right), \ldots,\left(P_{m}, u_{m}\right)\right)$.

In some GDM models, the importance degree of each expert is considered as known beforehand or provided by a reliable source while, in other cases, it is obtained in another way. For example, in [38], the authors suggest to give more importance to the experts that provide more consistent information. In such models, a consistency index is calculated for each expert by analyzing their individual FPRs, which are subsequently used to build the vector $U$. Many consistency measures have been defined so far for this purpose [40], although the most widely used are based on the concept of additive transitivity property.

In [9] the importance of each expert is calculated basing on data coming from a social network. The tie strength between pairs of experts is calculated through Social Network Analysis (SNA) techniques by combining the number of common connections with the number of direct interactions. Then the importance of an expert is obtained as the average strength of his ties with other experts.

In [11] and [12], the authors propose to use trust as a measure of experts' importance. Based on the history of past actions and behavior, trust reflects the actual reputation of an expert among his peers. Information about interpersonal trust is obtained by analyzing data coming from a type of social network in which the users explicitly express their opinion on other users as fuzzy trust statements. Trust statement related to each expert are aggregated and used to build the vector $U$. In particular, a linear combination of the consistency index and the trust degree of each expert is used to weight his FPR during the aggregation step.

\section{Social Influence and Opinion Change}

A GDM process, from the experts' point of view, only rarely results in expressing own opinions and accepting or rejecting the consensus to other's opinion. In real situations, individuals form their opinions in a complex interpersonal environment in which preferences on available alternatives are liable to change due to social influence.

In [9] social influence is defined as changes in individual's thoughts, feelings, attitudes or behaviors resulting from interaction with another individual or a group. In the same paper, the social influence among experts is calculated by combining the number of common connections with the number of direct interactions over a social network.

The use of data coming from social networks to support the decision making process is not new. In [41] SNA metrics are used to measure inter-organizational relationships with the aim of enhancing a decision making process for project selection. In [42] a consensus-based model based on SNA has been defined to reconcile conflicts in the collaborative annotation of media content. In [9] SNA metrics are used for the first time to estimate social influence between experts. The obtained value is then used to infer missing FPR values by combining values coming from influencing experts.

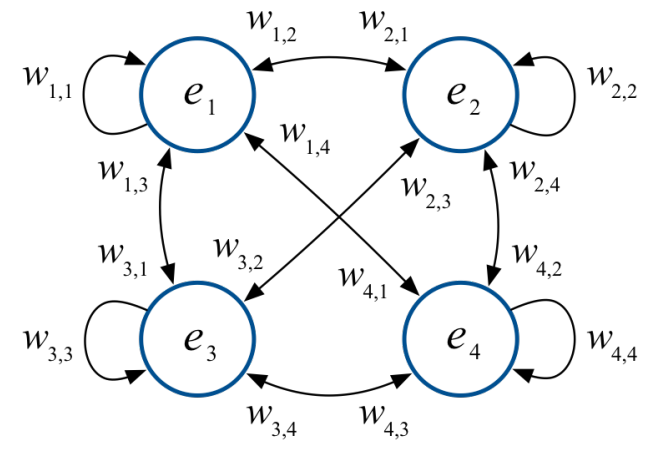

Fig 2. A SIN composed by 4-nodes

According to [6] and [7], influence can be modelled through a Social Influence Network (SIN): a directed graph between the set of experts $E$ and where each $\operatorname{arc}\left(e_{i}, e_{j}\right)$ has a weight $w_{i j} \in[0,1]$ that represents the strength of the influence of the $j$-th expert on the $i$-th one. Figure 2 shows an example of SIN. A SIN involving a set of experts $E=\left\{e_{1}, \ldots, e_{m}\right\}$ can be summarized by an $m \times m$ fuzzy adjacency matrix $W=\left(w_{i j}\right)$. In [6] it was suggested that the weights $w_{i 1}, \ldots, w_{i m}$ are directly chosen by the expert $e_{i}$ before he is informed of the preferences expressed by the others, on the basis of the relative importance he assigns to the opinion of the various experts, including himself. Selected weights must verify the normalization property $\sum_{j=1}^{m} w_{i j}=1$ for all $i \in\{1, \ldots, m\}$, i.e. the influences of peers on each expert sum to 1 .

If $y^{(1)}$ is an $m \times 1$ vector representing the initial experts' opinions on a given alternative, it is supposed that, after having interacted, this opinion vector will change to $y^{(2)}=W y^{(1)}$ due to interpersonal influence. If we suppose that each expert is informed that the others have changed their opinion, it is reasonable to expect that the expert will change again his opinion according to the same principle. By iterating the process, it is possible to obtain the experts' opinion after $t$ interactions as:

$$
y^{(t)}=W y^{(t-1)} .
$$

In [6] it was demonstrated that, if there exists a positive integer $t$ so that every element in at least one column of $W^{t}$ is positive, then the $m$ opinions are expected to converge to the same value. In [7] it was suggested to also specify the susceptibility of each expert $e_{i}$ to interpersonal influence as $a_{i i} \in[0,1]$. Let $y^{(1)}$ be the initial experts' opinions, after $t$ interactions, the updated opinions is obtained iteratively as:

$$
y^{(t)}=A W y^{(t-1)}+(I-A) y^{(1)}
$$

where $A=\operatorname{diag}\left(a_{11}, \ldots, a_{m m}\right)$ and $I$ is the $m \times m$ identity matrix. In other words, at each time, the current opinion of an expert is obtained as a linear combination of his initial opinion and the influenced opinion he had at the time immediately preceding. In [7] it was demonstrated that, if the matrix $I-A W$ is non-singular and the process reaches an equilibrium, i.e. $y^{(\infty)}=\lim _{t \rightarrow \infty} y^{(t)}$ exists, then:

$$
y^{(\infty)}=(I-A W)^{-1}(I-A) y^{(1)} .
$$


In [8], equations (11) and (12) were applied for the first time in a GDM process where the experts provide opinions on a set of alternatives $X=\left\{x_{1}, \ldots, x_{n}\right\}$ rather than on just one. For each expert $e_{i}$, the initial degree of preference $y_{i j}^{(1)}$ on each alternative $x_{j}$ is calculated starting from expert's individual FPR via the application of the QGDD metric to all preference values of the $j$-th row of the corresponding FPR.

Then, the influence model is applied on each column of the $m \times n$ matrix $Y^{(1)}=\left(y_{i j}^{(1)}\right)$ by extending equation (12) to matrices and obtaining: $Y^{(\infty)}=(I-A W)^{-1}(I-A) Y^{(1)}$. The $i$-th row of $Y^{(\infty)}$ represents the "influenced" preferences of the expert $e_{i}$ after having introjected his peers' opinions. The IIOWA operator is then applied to aggregate such influenced preferences.

\section{A New INFLuENCE-Guided FuzZy MODEL FOR GDM}

The proposed model is aimed at taking into account social influence within a GDM process both in general and in the presence of incomplete information. The research assumptions on which the proposed model is built are two: experts influence each other and the more an expert trusts in the capability of another expert, the more his opinion is influenced by the trusted expert. This has immediate applications in presence of incomplete information i.e. when experts cannot express an opinion on any of the available alternatives. Trust statements collected among experts are used to initialize a SIN that is in turn employed to improve provided preferences and to estimate the missing ones.

To make the model immediately applicable in practice, we have chosen fuzzy rankings (defined in [43]) as the representation format for preferences expression since they are user friendly and less vulnerable to inconstancy than FPRs. The same representation format is used for both the opinions on alternatives and the trust statements on experts.

Given a set of experts $E=\left\{e_{1}, \ldots, e_{m}\right\}$ and a set of alternatives $X=\left\{x_{1}, \ldots, x_{n}\right\}$, the proposed model works through the following steps:

1. opinions collection: each expert $e_{i} \in E$ specifies his preferences on alternatives in $X$ through a (possibly incomplete) fuzzy ranking $R_{i}$;

2. trust statements collection: each expert $e_{i} \in E$ specifies the trust he has in the opinion of experts belonging to $E$ (including himself) through a (possibly incomplete) fuzzy ranking $R_{i}^{e}$;

3. fuzzy ranking conversion: fuzzy rankings $R_{i}$ and $R_{i}^{e}$ are converted to (possibly incomplete) individual FPRs $P_{i}$ and $P_{i}^{e}$ for $i \in\{1, \ldots, m\}$;

4. social influence network generation: the FPRs $P_{i}^{e}$ for $i \in\{1, \ldots, m\}$, representing degree of trust between experts, are used to generate a SIN characterised by the $m \times m$ fuzzy adjacency matrix $W$;

5. missing preferences estimation: individual FPRs $P_{i}$ for $i \in\{1, \ldots, m\}$, in presence of missing information, are completed by injecting values from other FPRs via $W$;

6. influence-guided preferences evolution: to simulate the

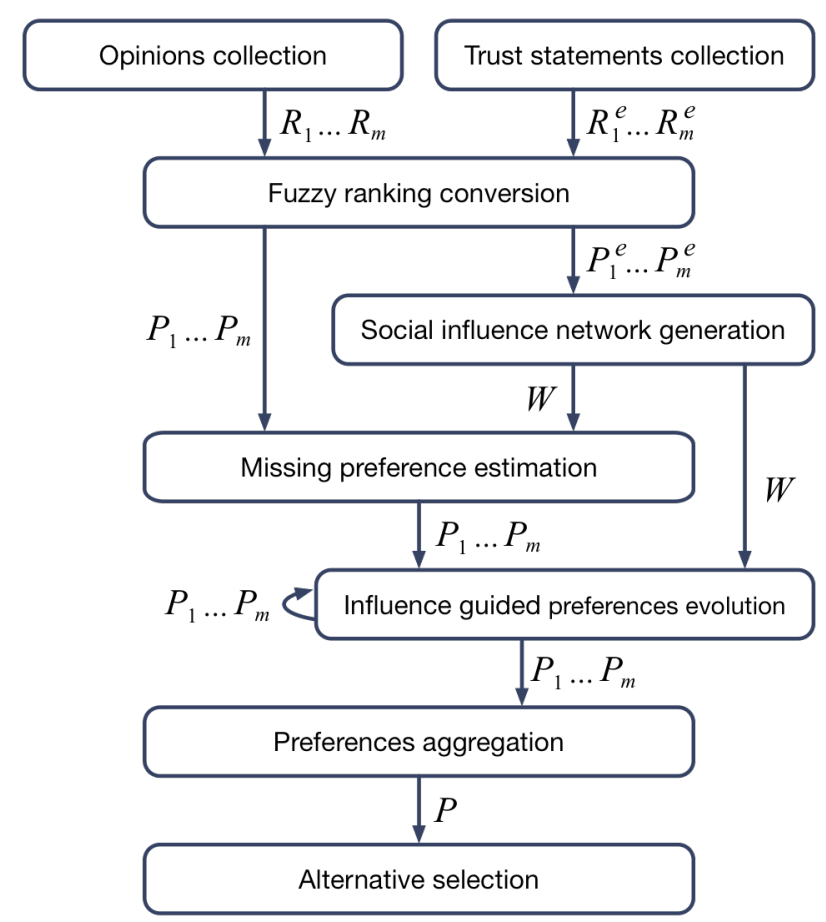

Fig 3. Model steps and information flow

effects of experts' interpersonal influence, the completed individual FPRs $P_{i}$ for $i \in\{1, \ldots, m\}$ are updated via $W$ until convergence;

7. preferences aggregation: the updated individual FPRs $P_{i}$ for $i \in\{1, \ldots, m\}$ are aggregated through OWA to obtain the collective FPR $P$.

8. alternative selection: the dominance degree $\phi\left(x_{i}\right)$ is estimated for each alternative $x_{i} \in X$ according to $P$, then alternatives are ranked from the best to the worst and the first one is selected.

The information flow among the described steps is summarized in Figure 3 while the next subsections provide details on each step. In particular subsection III.A deals with the collection of opinions and trust statements and their conversion to FPRs (steps 1-3); subsection III.B explains how the SIN is generated and applied to estimate missing preferences (steps 4-5); subsection III.C deals with the application of the influence model on obtained FPRs, their aggregation and alternative selection (steps 6-8).

\section{A. Preferences Collection and FPRs Generation}

Although FPRs are among the most commonly used methods to express preferences on different alternatives, they are not free from drawbacks. First of all, especially when dealing with a large number of alternatives, the definition of a FPRs may become complex and time-consuming. Moreover, while they allow to focus on only two options at a time, this can let the expert lose the global perception of the problem with the risk of introducing inconsistency that subsequently could impact negatively on the decision process. For these reasons, alternative representation formats of preferences are often adopted in real GDM settings and, if necessary, transformation functions are applied to obtain consistent FPRs. 
A fair compromise between expressiveness and easiness is represented by fuzzy rankings as defined in [43] where experts rank the available alternatives and express the grade to which each alternative is better than the subsequent compared one. A fuzzy ranking can be represented through a finite sequence $R=\left(\begin{array}{llll}x_{\sigma(1)} s_{1} & x_{\sigma(2)} & \ldots & x_{\sigma(k-1)} s_{k-1} \\ x_{\sigma(k)}\end{array}\right)$ with $k \leq n$. Terms in odd positions in the sequence represent a subset of the alternatives, while $\sigma:\{1, \ldots n\} \rightarrow\{1, \ldots k\}$ is a $k$-permutation function. Terms in even positions belong to the set of symbols $S=\{\gg,>, \geq, \approx\}$ and define a degree of preference between subsequent terms (with $\gg$ meaning "is much better than", $>$ "is better than", $\geq$ "is a little better than" and $\approx$ "is similar to"). Each alternative appears at most once in the ranking so cycles are not allowed although partial rankings are admitted. Inspired by studies on the use of linguistic labels in GDM like [44], the cardinality of $S$ has been chosen small enough so as not to impose useless precision to the experts and rich enough to allow a discrimination of the relative performance of the alternatives. On the other hand, the possibility to compose fuzzy rankings by chaining alternatives and symbols, allows to indirectly express a wide variety of preferences levels.

Starting from a fuzzy ranking it is possible to generate a FPR as defined in [43] and [45], where a fuzzy preference degree is associated to each symbol of $S$. Unfortunately, doing so the generated FPR is not guaranteed to be additive consistent. For this reason, we propose a new transformation function based on the one defined in [26] which leads to additive consistent FPRs. A relative strength $|\mathrm{s}|$ is associated to each symbol $s \in S$, where $|\gg|=2,|>|=1,|\geq|=0.5$ and $|\approx|=0$ and, given a fuzzy ranking $R$, a crispy rank $r\left(x_{i}\right)$ is associated to each alternative so that:

- $r\left(x_{\sigma(1)}\right)=1$;

- $r\left(x_{\sigma(i)}\right)=r\left(x_{\sigma(i-1)}\right)+\left|s_{i-1}\right| \forall i \in\{2, \ldots, k\} ;$

- $\quad r\left(x_{i}\right)$ is undefined if $\sigma(i)$ is undefined i.e. if the $i$ th alternative does not appear in $R$.

Then, for any pair of alternatives $x_{i}$ and $x_{j}$ present in a fuzzy ranking $R$, the following preference value can be defined:

$$
p_{i j}=\frac{1}{2}\left(1+\frac{r\left(x_{j}\right)-r\left(x_{i}\right)}{r \max -1}\right)
$$

where $\operatorname{rmax}=r\left(x_{\sigma(k)}\right)$ is the maximum rank. The special case $r \max =1$, occurring when an expert considers all alternatives in the fuzzy ranking equally in terms of preference, is handled by setting $p_{i j}=0.5$. It is easy to demonstrate that the existing elements of the obtained FPR $P$ verify the additive consistency property (see appendix).

It should be noted that the relative strength of each symbol has been selected so that, apart from $\approx$, each symbol doubles the relative strength of the next one. By only using the symbol $>$, the fuzzy ranking becomes a simple ordering of alternatives and equations (13) and (14) become the same defined in [24]. The use of the symbols $\gg$ or $\geq$ in place of $>$ respectively doubles or halves the distance of the preceding and subsequent terms in the ranking while the use of $\approx$ means that the preced- ing and subsequent terms have the same rank.

As an option, experts may be allowed to provide a set of fuzzy rankings, interesting disjoint subsets of $X$, rather than just one. In this way it is possible to deal with the case in which an expert judges some options as mutually incomparable. To obtain an additive consistent FPR even in this case, it is enough to iterate equation (13) on any provided fuzzy ranking.

Fuzzy rankings are used in our model to let experts express their opinion with respect to (a subset of) the alternatives as well as their trust on (a subset of) the experts. More formally, each expert $e_{k} \in E$ provides a fuzzy ranking $R_{k}$ on the set of alternatives in $X$ and a fuzzy ranking $R_{k}^{e}$ on the set of experts $E$ (including himself).

Starting from $R_{k}$ and $R_{k}^{e}$, by applying equation (14), the corresponding (incomplete) FPRs $P_{k}$ and $P_{k}^{e}$ are computed and taken forward to the next steps. Being $m$ the number of experts and $n$ the number of alternatives, the time complexity of this step is $\mathcal{O}\left(m \cdot n^{2}\right)$. To clarify the concept, consider the following example.

Example 1. Let us suppose that we have a set of five alternatives $X=\left\{x_{1}, x_{2}, x_{3}, x_{4}, x_{5}\right\}$ and a set of three experts $E=\left\{e_{1}, e_{2}, e_{3}\right\}$, that expert $e_{1}$ provides the fuzzy ranking of alternatives: $R_{1}=x_{4} \gg x_{5} \approx x_{2} \geq x_{1}$ and the fuzzy ranking of experts: $R_{1}^{e}=e_{2} \gg e_{1} \approx e_{3}$. In $R_{1}$ the expert states that the alternative $x_{4}$ is much better than $x_{5}$ which in turn is similar to $x_{2}$. The latter in turn is a little better than $x_{1}$. Moreover he has no opinion at all on $x_{3}$. In $R_{1}^{e}$ the expert states that he thinks that the expert $e_{2}$ is much more trustable than $e_{1}$ (himself) who in turn is trustable as $e_{3}$. Starting from $R_{1}$ and $R_{1}^{e}$, by applying equations (13)-(14), the following associated FPRs are obtained:

$$
P_{1}=\left(\begin{array}{ccccc}
0.5 & 0.4 & - & 0 & 0.4 \\
0.6 & 0.5 & - & 0.1 & 0.5 \\
- & - & - & - & - \\
1 & 0.9 & - & 0.5 & 0.9 \\
0.6 & 0.5 & - & 0.1 & 0.5
\end{array}\right) ; P_{1}^{e}=\left(\begin{array}{ccc}
0.5 & 0 & 0.5 \\
1 & 0.5 & 1 \\
0.5 & 0 & 0.5
\end{array}\right)
$$

\section{B. Using Social Influence to Estimate Missing Preferences}

When some experts express their opinions only on a subset of available alternatives, incomplete FPRs (i.e. FPRs with some undefined values) are generated through equations (13)-(14). In particular, if the $i$-th alternative does not appear in a given fuzzy ranking $R$, then both the $i$-th row and the $i$-th column of the corresponding FPR $P$ remains undefined (e.g. alternative $x_{3}$ in example 1). As seen in II.A, this is considered an ignorance situation that can be solved by selecting seed values to initialize the missing preferences and by iterating the equations (6)-(7) until the convergence is reached and the final estimates obtained.

Several methods have been proposed so far to obtain seed values (as described in II.A). Here we propose to obtain seed values from preferences provided by the experts that are trusted by the one whose FPR has to be completed. This is to say that, when an expert is asked to evaluate an unknown alternative, he forms his judgment using the opinion of experts he trusts. 
To do that, we firstly generate a SIN starting from the opinions on experts collected in $P_{k}^{e}$ for $k \in\{1, \ldots, m\}$. Then, any missing preference in $P_{k}$ for $k \in\{1, \ldots, m\}$ is estimated according to the generated SIN.

As explained in II.C, a SIN is characterized by a fuzzy adjacency matrix $W=\left(w_{k l}\right)$ where each element $w_{k l} \in[0,1]$ represents the strength of the influence of the $l$-th expert on the $k$ th one for $k, l \in\{1, \ldots, m\}$. So, the elements of $k$-th row of $W$ can be obtained from $P_{k}^{e}$ through FPR measures defined in section II like $Q G D D$. To comply the SIN property so that $\sum_{i=1}^{m} w_{k i}=1$, a normalization step is needed as follows:

$$
w_{k l}=\frac{\phi_{k}\left(e_{l}\right)}{\sum_{i=1}^{m} \phi_{k}\left(e_{i}\right)}
$$

and:

$$
\phi_{k}\left(e_{l}\right)=O W A_{Q}\left(p_{k l j}^{e} ; j=1, \ldots, m: p_{k l j}^{e} \text { is defined }\right) .
$$

Undefined elements of $P_{k}^{e}$ are not considered in equation (17); when the $l$-th row of $P_{k}^{e}$ is undefined (i.e. when $e_{k}$ expresses no preferences on $\left.e_{l}\right) \phi_{k}\left(e_{l}\right)=0$; in the special case which the $k$-th expert only trusts himself, we obtain via equations (16)-(17): $w_{k l}=0$ for $k \neq l$ and $w_{k k}=1$ meaning that the expert is not influenced by any other.

Based on the generated SIN, a missing preference $p_{k i j}$ of an FPR $P_{k}$ coming from $e_{k}$ is estimated through the I-IOWA operator (defined in II.A) where the preferences to aggregate come from all the defined FPRs $P_{l}$ with $l \in\{1, \ldots, m\}$ while the importance degrees, which represent the trust degree of $e_{k}$ on each expert of $E$, come from $W$. Basing on equation (8), a missing preference $p_{k i j}$ is so estimated as follows:

$$
\varepsilon\left(p_{k i j}\right)=I-I O W A_{Q}\left(\left(p_{l i j}, w_{k l}\right) ; l=1, \ldots, m: p_{l i j} \text { is defined }\right)
$$

Undefined elements of $P_{l}$ with $l \in\{1, \ldots, m\}$ are not considered in equation (18). If seed values for some preferences are still missing (e.g. when the same preferences are missing in the FPRs of any trusted expert), then the estimation process based on equation (18) is repeated on FPRs injected with estimated values. The process is re-iterated until no additional seed values can be calculated. Then, the final estimates are computed through the iterative application of equations (6)-(7) until convergence is reached.

In some cases it is possible that some FPR still remain partially undefined. Given an FPR $P_{k}$ and an alternative $x_{i} \in X$, when none of the experts (directly or indirectly) trusted by $e_{k}$ have an opinion on $x_{i}$ i.e. when $p_{l i j}$ and $p_{l j i}$ are undefined for any $j \in\{1, \ldots, n\}$ and any $l$ so that a path (that excludes 0 weighted arcs) from $e_{l}$ to $e_{k}$ exists in the SIN, then both the $i$ th row and the $i$-th column of $P_{k}$ remain undefined. In case the SIN is a connected graph this means that all experts have no opinion on $x_{i}$. This suggests that the alternative is of no interest for the whole group so it can be removed from $X$. Conversely, in case the SIN is disconnected, it is possible that other (untrusted) experts have provided an opinion on $x_{i}$. In such cases $x_{i}$ can't be removed and remaining undefined FPRs elements must be estimated through a different method among those discussed in II.A (e.g. through indifference by setting the seed value to 0.5 ).

Assuming that OWA and I-IOWA operators use state-of-theart sorting algorithms, the overall time complexity of the SIN generation step is $\mathcal{O}\left(m \cdot n^{2} \log n\right)$. Instead, the time complexity of the preference estimation step is affected by the number of missing preferences and can be asymptotically limited by $\Omega\left(m \cdot n^{2}\right)$ and $\mathcal{O}\left(m \cdot n^{3} \log n\right)$.

Example 2. Let $E, X, P_{1}$ and $P_{1}^{e}$ be as reported in example 1, by applying equations (16)-(17) with values from $P_{1}^{e}$ and using the fuzzy quantifier $(0,1)$ corresponding to the linguistic label "much" (see Figure 1) to guide the OWA operator, the obtained weights are: $w_{1,1}=0.17 ; w_{1,2}=0.17 ; w_{1,3}=0.66$; meaning that, to complete $P_{1}$, values from $P_{2}$ are weighted 0.17 while values from $P_{3}$ are weighted 0.66 (the first weight is irrelevant given that it refers to expert self-trust). Let suppose that the experts $e_{2}$ and $e_{3}$ specify the fuzzy rankings: $R_{2}$ $=x_{4} \approx x_{5}>x_{3}>x_{2}$ and $R_{3}=x_{3} \approx x_{5} \geq x_{4} \gg x_{1}$. The corresponding FPRs, obtained through equations (13)-(14), are:

$$
\begin{aligned}
P_{2} & =\left(\begin{array}{ccccc}
- & - & - & - & - \\
- & 0.50 & 0.25 & 0 & 0 \\
- & 0.75 & 0.50 & 0.25 & 0.25 \\
- & 1 & 0.75 & 0.50 & 0.50 \\
- & 1 & 0.75 & 0.50 & 0.50
\end{array}\right) ; \\
P_{3} & =\left(\begin{array}{ccccc}
0.50 & - & 0 & 0.10 & 0 \\
- & - & - & - & - \\
1 & - & 0.50 & 0.60 & 0.50 \\
0.90 & - & 0.40 & 0.50 & 0.40 \\
1 & - & 0.50 & 0.60 & 0.50
\end{array}\right) .
\end{aligned}
$$

Seed values for missing preferences of $P_{1}$ are generated from $P_{2}, P_{3}$ and $w_{k}$, via equation (18) and using the quantifier $(0,1)$ to guide the I-IOWA operator: $\varepsilon\left(p_{113}\right)=0, \varepsilon\left(p_{123}\right)=0.25$, $\varepsilon\left(p_{131}\right)=1, \varepsilon\left(p_{132}\right)=0.75, \varepsilon\left(p_{133}\right)=0.5, \varepsilon\left(p_{134}\right)=0.32$, $\varepsilon\left(p_{135}\right)=0.3, \varepsilon\left(p_{143}\right)=0.68, \varepsilon\left(p_{153}\right)=0.7$. By iteratively applying equations (6)-(7) until convergence and injecting the last estimations in $P_{1}$, the FPR coming from $e_{1}$ is completed as follows (where injected values are reported in bold):

$$
P_{1}=\left(\begin{array}{ccccc}
0.5 & 0.4 & \mathbf{0 . 1 9} & 0 & 0.4 \\
0.6 & 0.5 & \mathbf{0 . 3 2} & 0.1 & 0.5 \\
\mathbf{0 . 8 1} & \mathbf{0 . 6 8} & \mathbf{0 . 5} & \mathbf{0 . 2 8} & \mathbf{0 . 5 9} \\
1 & 0.9 & \mathbf{0 . 7 2} & 0.5 & 0.9 \\
0.6 & 0.5 & \mathbf{0 . 4 1} & 0.1 & 0.5
\end{array}\right) .
$$

\section{Preferences Evolution and Best Alternative Selection}

To simulate the effects of interpersonal influence among experts, the individual FPRs obtained at the preceding steps are revised using the SIN generated with equations (16)-(17). The aim is to predict the final decision that will be adopted by the group of experts as a result of interaction, without the need to actually perform such interaction. To do that we apply an iterative process like that described in section II.C where at each step the individual FPR of each of the experts slightly changes to take into account the influence coming from trusted experts. Differently from [8] in our model the influence model directly impacts individual FPRs rather than utility vectors obtained 
from them. Being $P_{k}^{(1)}=\left(p_{k i j}^{(1)}\right)$ the FPR representing the initial opinion of the $k$-th expert with $k \in\{1, \ldots, m\}$ and $i, j \in\{1, \ldots, n\}$, it is possible to estimate the elements of the $k$ th expert's FPR after $t$ interactions based on the SIN fuzzy adjacency matrix $W$ as follows:

$$
p_{k i j}^{(t)}=I-I O W A_{Q}\left(\left(p_{1 i j}^{(t-1)}, w_{k 1}\right), \ldots,\left(p_{m i j}^{(t-1)}, w_{k m}\right)\right) .
$$

In other words, at each step, each preference is updated by composing the current preference with preferences coming from all the experts via the I-IOWA operator. The importance degree of each contribution matches the strength of the interpersonal influence coming from $W$. Extending the notation to matrices, we can rewrite equation (21) as follows:

$$
P_{k}^{(t)}=I-I O W A_{Q}\left(\left(P_{1}^{(t-1)}, w_{k 1}\right), \ldots,\left(P_{m}^{(t-1)}, w_{k m}\right)\right) .
$$

When the fuzzy quantifier $Q=(0,1)$, corresponding to the linguistic label "much" (see Figure 1), is used to obtain the IIOWA weights, it can be demonstrated that, if there exists a positive integer $l$ so that every element in at least one column of $W^{l}$ is positive, then the $m$ FPRs are expected to converge to the same FPR (see appendix). In practical applications the process may be stopped after a fixed number of iteration or when the average absolute difference between FPRs values in two subsequent steps is under a given threshold $\theta$ i.e. when:

$$
\frac{1}{m \cdot n^{2}} \sum_{1 \leq i, j \leq n ; 1 \leq k \leq m}\left|p_{k i j}^{(t)}-p_{k i j}^{(t-1)}\right| \leq \theta
$$

When the stopping conditions are met, in case of lack of convergence, the obtained FPRs are aggregated through the $O W A_{Q}$ operator defined in section II, whose weights are initialized according to equation (4). A score value $\phi\left(x_{i}\right)$ is then calculated for each $x_{i} \in X$ through the QGDD operator defined by equation (5) and the best alternative is chosen as the result of the GDM problem.

To obtain a more exhaustive and easy to understand solution to the problem, it is possible to convert the obtained score values $\phi\left(x_{i}\right)$ for $i \in\{1, \ldots, n\}$, back to a collective fuzzy ranking of alternatives $R=\left(\begin{array}{llll}x_{\sigma(1)} s_{1} x_{\sigma(2)} & \ldots & x_{\sigma(n-1)} s_{n-1} x_{\sigma(n)}\end{array}\right)$ where $s_{i} \in\{\gg,>, \geq, \approx\}$ and $\sigma:\{1, \ldots n\} \rightarrow\{1, \ldots n\}$ is a permutation function such that $\phi\left(x_{\sigma(i)}\right) \geq \phi\left(x_{\sigma(i+1)}\right)$ for $i \in\{1, \ldots, n-1\}$. Basing on the relative strength associated to each symbol (as defined in A), it is possible to obtain any $s_{i}$ for $i \in\{1, \ldots, n\}$ as follows:

$$
s_{i}=\left\{\begin{array}{l}
\approx \text { if } \phi\left(x_{\sigma(i+1)}\right)-\phi\left(x_{\sigma(i)}\right)<0.25 \cdot \delta \\
\geq \text { if } 0.25 \cdot \delta \leq \phi\left(x_{\sigma(i+1)}\right)-\phi\left(x_{\sigma(i)}\right)<0.75 \cdot \delta \\
>\text { if } 0.75 \cdot \delta \leq \phi\left(x_{\sigma(i+1)}\right)-\phi\left(x_{\sigma(i)}\right)<1.5 \cdot \delta \\
\gg \text { if } 1.5 \cdot \delta \leq\left(x_{\sigma(i+1)}\right)-\phi\left(x_{\sigma(i)}\right)
\end{array}\right.
$$

where $\delta$ is the average difference between two subsequent ranked score values:

$$
\delta=\frac{1}{n-1} \sum_{i=1}^{n-1}\left(\phi\left(x_{\sigma(i+1)}\right)-\phi\left(x_{\sigma(i)}\right)\right)
$$

The time complexity of each iteration of the preference evolution step is $\mathcal{O}\left(m \cdot n^{3} \log n\right)$. Being the number of iterations limited by a constant, it can be considered as asymptotically negligible. The aggregation of FPRs (in case of lack of convergence) has a time complexity of $\mathcal{O}\left(m \cdot n^{3} \log n\right)$, while the complexity of the alternative selection step is $\mathcal{O}\left(n^{2} \log n\right)$.

Example 3. Let $E, X, P_{2}, P_{3}$ and $P_{1}^{e}$ be as reported in previous examples and the completed individual FPR $P_{1}$ as reported in equation (20). Let $R_{2}^{e}=e_{1} \approx e_{2} \gg e_{3}$ and $R_{3}^{e}=e_{3}>e_{2}>e_{1}$ be the fuzzy rankings of experts defined, respectively, by $e_{2}$ and $e_{3}$, through equations (16)-(17) it is possible to obtain the SIN represented by the following matrix:

$$
W=\left(\begin{array}{ccc}
0.17 & 0.67 & 0.17 \\
0.5 & 0.5 & 0 \\
0.08 & 0.33 & 0.85
\end{array}\right)
$$

Using $W$, it is possible to estimate the completed individual FPRs $P_{2}$ and $P_{3}$ through equation (18) as follows (where injected values are represented in bold):

$$
\begin{aligned}
P_{2} & =\left(\begin{array}{ccccc}
\mathbf{0 . 5} & \mathbf{0 . 5 6} & \mathbf{0 . 3 4} & \mathbf{0 . 7 7} & \mathbf{0 . 1 6} \\
\mathbf{0 . 3 8} & 0.5 & 0.25 & 0 & 0 \\
\mathbf{0 . 5 2} & 0.75 & 0.5 & 0.25 & 0.25 \\
\mathbf{0 . 8 6} & 1 & 0.75 & 0.5 & 0.5 \\
\mathbf{0 . 7 8} & 1 & 0.75 & 0.5 & 0.5
\end{array}\right) ; \\
P_{3} & =\left(\begin{array}{ccccc}
0.5 & 0.41 & 0 & 0.1 & 0 \\
0.59 & 0.5 & 0.12 & 0.16 & 0.09 \\
1 & 0.88 & 0.5 & 0.6 & 0.5 \\
0.9 & 0.84 & 0.4 & 0.5 & 0.4 \\
1 & 0.91 & 0.5 & 0.6 & 0.5
\end{array}\right)
\end{aligned}
$$

The completed FPRs are then updated according to equation (22) simulating the effect of influence. The fuzzy quantifier $Q=(0,1)$, corresponding to the linguistic label "much", is used to guide the I-IOWA operator. The following matrices represent the evolution of $P_{1}$ after 2 and 6 iterations:

$$
\begin{aligned}
P_{1}^{(2)} & =\left(\begin{array}{ccccc}
0.5 & 0.51 & 0.26 & 0.07 & 0.17 \\
0.45 & 0.5 & 0.24 & 0.04 & 0.1 \\
0.65 & 0.76 & 0.5 & 0.31 & 0.35 \\
0.89 & 0.56 & 0.69 & 0.5 & 0.55 \\
0.78 & 0.9 & 0.65 & 0.45 & 0.5
\end{array}\right) ; \\
P_{1}^{(6)} & =\left(\begin{array}{ccccc}
0.5 & 0.48 & 0.25 & 0.05 & 0.22 \\
0.48 & 0.5 & 0.26 & 0.05 & 0.18 \\
0.68 & 0.74 & 0.5 & 0.31 & 0.4 \\
0.91 & 0.95 & 0.69 & 0.5 & 0.62 \\
0.75 & 0.82 & 0.6 & 0.38 & 0.5
\end{array}\right) .
\end{aligned}
$$

After 6 iterations all individual FPRs converge to the same FPR $P=P_{1}^{(6)}=P_{2}^{(6)}=P_{3}^{(6)}$ that can be considered as the collective preference relation of consensus (so there is no need for aggregation). By applying equation (5) the score values associated to alternatives are: $\phi\left(x_{1}\right)=0.25 ; \phi\left(x_{2}\right)=0.24$; $\phi\left(x_{3}\right)=0.53 ; \phi\left(x_{4}\right)=0.79 ; \phi\left(x_{5}\right)=0.64$. The best alternative is $x_{4}$ which can be considered the solution of the problem. By applying equations (24) and (25) it is also possible to obtain the following collective fuzzy ranking of alternatives: $x_{4}>x_{5}>x_{3} \gg x_{1} \approx x_{2}$. 
TABLE I

COLLECTED FUZZY RANKINGS OF ALTERNATIVES AND EXPERTS (LEFT) AND COMPLETED FUZZY RANKINGS OF ALTERNATIVES (RIGHT) - FIRST CASE

\begin{tabular}{ccc|c}
\hline \hline Expert & Fuzzy rankings of alternatives & Fuzzy rankings of experts & Completed fuzzy rankings of alternatives \\
\hline$e_{1}$ & $x_{5} \gg x_{7} \approx x_{8} \geq x_{1} \approx x_{3}>x_{4} \gg x_{2}$ & $e_{2} \gg e_{1}>e_{4} \geq e_{5}$ & $x_{5}>x_{6} \geq x_{10}>x_{7} \approx x_{8} \geq x_{1} \geq x_{3} \gg x_{4} \gg x_{9}>x_{2}$ \\
$e_{2}$ & $x_{10} \approx x_{6}>x_{2} \geq x_{1} \gg x_{3} \geq x_{9} \approx x_{5}$ & $e_{3}>e_{2} \approx e_{4} \geq e_{5}>e_{6}$ & $x_{10} \geq x_{6} \gg x_{2}>x_{1}>x_{4} \gg x_{3} \geq x_{8} \geq x_{5} \geq x_{7} \approx x_{9}$ \\
$e_{3}$ & $x_{3} \approx x_{5}>x_{10} \gg x_{1}>x_{2}>x_{6} \approx x_{7} \approx x_{8}$ & $e_{3} \gg e_{6} \geq e_{2}>e_{5}$ & $x_{3} \approx x_{5}>x_{10} \gg x_{4} \geq x_{1} \gg x_{2} \gg x_{6} \approx x_{7} \approx x_{8} \geq x_{9}$ \\
$e_{4}$ & $x_{6}>x_{2} \geq x_{1}>x_{9} \approx x_{5}>x_{8}$ & $e_{4}>e_{3}>e_{2} \approx e_{1}>e_{5} \approx e_{6}$ & $x_{10} \geq x_{6}>x_{3}>x_{2} \geq x_{1} \geq x_{4} \gg x_{5} \geq x_{7} \geq x_{9} \gg x_{8}$ \\
$e_{5}$ & $x_{3}>x_{5} \gg x_{8}>x_{1}>x_{10}>x_{6}>x_{2}$ & $e_{3} \geq e_{5} \geq e_{6}>e_{1} \approx e_{2}$ & $x_{3}>x_{5} \gg x_{8}>x_{1} \approx x_{4}>x_{10} \gg x_{6} \geq x_{7}>x_{2} \geq x_{9}$ \\
$e_{6}$ & $x_{10} \approx x_{4}>x_{5} \gg x_{6}>x_{2}$ & $e_{6} \gg e_{2} \geq e_{5}>e_{4}$ & $x_{10}>x_{4}>x_{5} \gg x_{1} \approx x_{3}>x_{8} \geq x_{6}>x_{7}>x_{9} \geq x_{2}$ \\
\hline \hline
\end{tabular}

\section{EXPERIMENTS AND EVALUATION}

This section describes two in silico experiments of the proposed methodology aimed at illustrating its operational steps and its convergence properties. Eventually, a qualitative comparison with other existing methods is proposed.

\section{A. First Case: Convergence of Experts' Opinions}

Let $E=\left\{e_{1}, e_{2}, e_{3}, e_{4}, e_{5}, e_{6}\right\}$ be a set of experts that have to choose the best alternative among those available in the set $X=\left\{x_{1}, x_{2}, x_{3}, x_{4}, x_{5}, x_{6}, x_{7}, x_{8}, x_{9}, x_{10}\right\}$. According to the defined model, experts use fuzzy rankings to express both their preferences on alternatives and their trust on other experts. Defined fuzzy rankings are reported in the second and third columns of Table I. As it can be seen, many experts provide incomplete information both with respect to alternatives and to other experts. For example $e_{1}$ just evaluates 7 alternatives over 10 and express his trust on 4 experts over 6.

Applying equations (13)-(14), the fuzzy rankings on alternatives are converted into FPRs (see Table II). As it can be seen, many elements remain undefined given the incompleteness of experts' opinion.

The same process is repeated with fuzzy rankings of experts and obtained FPRs (that are not reported for reasons of brevity) are, in turn, used to build an SIN via equations (16)-(17). It is important to note that, even if information on trust is incomplete, the SIN generation process is able to initialize any SIN weight. The obtained SIN, shown in Figure 4, can be summarized by the following fuzzy adjacency matrix:

$$
W=\left(\begin{array}{cccccc}
0.26 & 0.45 & 0 & 0.17 & 0.12 & 0 \\
0 & 0.22 & 0.32 & 0.22 & 0.17 & 0.07 \\
0 & 0.20 & 0.44 & 0 & 0.11 & 0.25 \\
0.16 & 0.16 & 0.22 & 0.29 & 0.09 & 0.09 \\
0.09 & 0.09 & 0.34 & 0 & 0.28 & 0.21 \\
0 & 0.25 & 0 & 0.11 & 0.20 & 0.44
\end{array}\right) .
$$

Applying the process described in section III.B it is then possible to estimate missing preferences by injecting external seeds from trusted experts (according to the SIN) and consolidate them through harmonization with existing preferences using the additive consistency property process described in section II.A. Completed FPRs are reported in Table III. To make these results more readable, we apply equations (24)(25) to obtain back the completed fuzzy rankings after the in- jection of external preferences. They are reported in the last column of Table I for comparison with the initial rankings.

The next step consists in executing the process described in section III.C to let experts' preferences evolve according to social influence. The process is expected to converge since at least one column of $W$ has all positive elements. In fact, after 5 iterations, the experts' preferences converge to the same collective FPR $P$ reported below:

$P=\left(\begin{array}{cccccccccc}0.5 & 0.58 & 0.4 & 0.39 & 0.44 & 0.51 & 0.59 & 0.61 & 0.67 & 0.36 \\ 0.4 & 0.5 & 0.31 & 0.24 & 0.32 & 0.43 & 0.48 & 0.51 & 0.59 & 0.23 \\ 0.57 & 0.67 & 0.5 & 0.46 & 0.51 & 0.61 & 0.69 & 0.71 & 0.71 & 0.44 \\ 0.46 & 0.67 & 0.38 & 0.5 & 0.46 & 0.6 & 0.56 & 0.58 & 0.63 & 0.37 \\ 0.54 & 0.68 & 0.46 & 0.45 & 0.5 & 0.6 & 0.67 & 0.69 & 0.68 & 0.42 \\ 0.47 & 0.57 & 0.37 & 0.31 & 0.4 & 0.5 & 0.53 & 0.57 & 0.66 & 0.29 \\ 0.3 & 0.42 & 0.2 & 0.21 & 0.23 & 0.36 & 0.5 & 0.42 & 0.45 & 0.17 \\ 0.35 & 0.46 & 0.25 & 0.26 & 0.29 & 0.4 & 0.46 & 0.5 & 0.5 & 0.24 \\ 0.25 & 0.35 & 0.2 & 0.17 & 0.25 & 0.27 & 0.35 & 0.38 & 0.5 & 0.12 \\ 0.61 & 0.77 & 0.53 & 0.53 & 0.58 & 0.71 & 0.73 & 0.73 & 0.8 & 0.5\end{array}\right)$

From $P$ by applying equation (5) to calculate the dominance degrees: $\phi\left(x_{1}\right)=0.51, \quad \phi\left(x_{2}\right)=0.39, \quad \phi\left(x_{3}\right)=0.6$, $\phi\left(x_{4}\right)=0.52, \phi\left(x_{5}\right)=0.58, \phi\left(x_{6}\right)=0.46, \phi\left(x_{7}\right)=0.31$, $\phi\left(x_{8}\right)=0.36, \phi\left(x_{9}\right)=0.26, \phi\left(x_{1}\right)=0.67$. So, the best alternative is $x_{10}$. In addition, the obtained dominance degrees can be used to generate the following ranking of alternatives:

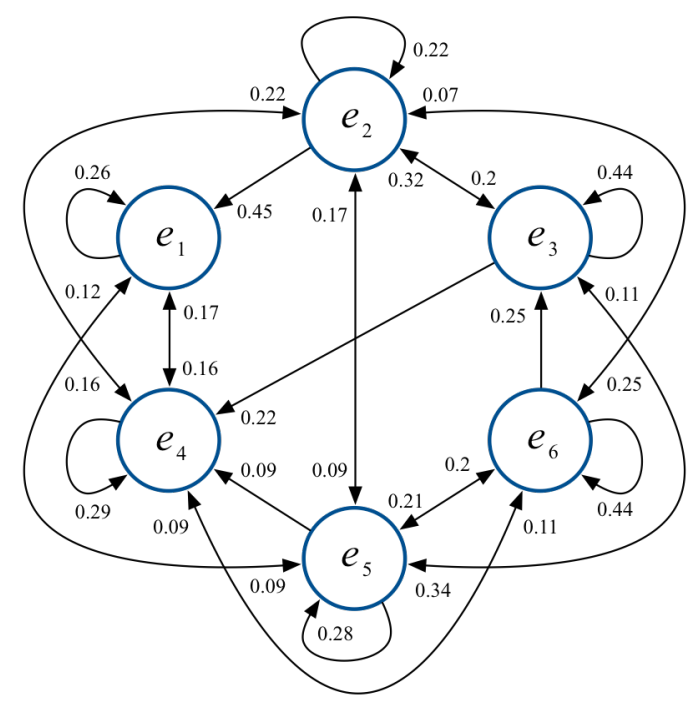

Fig 4. The generated SIN - first case 
TABLE II

EXPERTS’ INITIAL OPINIONS CONVERTED IN FPRS - FIRST CASE

\begin{tabular}{|c|c|c|c|c|c|c|c|c|c|c|c|c|c|c|c|c|c|c|c|c|c|}
\hline \multirow{10}{*}{$P_{1}=$} & 0.50 & 0.77 & 0.50 & 0.59 & 0.27 & - & 0.45 & 0.45 & - & - & \multirow{10}{*}{$P_{2}=$} & 0.50 & 0.44 & 0.75 & - & 0.81 & 0.31 & - & - & 0.81 & 0.31 \\
\hline & 0.23 & 0.50 & 0.23 & 0.32 & 0.00 & - & 0.18 & 0.18 & - & - & & 0.56 & 0.50 & 0.81 & - & 0.88 & 0.38 & - & - & 0.88 & 0.38 \\
\hline & 0.50 & 0.77 & 0.50 & 0.59 & 0.27 & - & 0.45 & 0.45 & - & - & & 0.25 & 0.19 & 0.50 & - & 0.56 & 0.06 & - & - & 0.56 & 0.06 \\
\hline & 0.41 & 0.68 & 0.41 & 0.50 & 0.18 & - & 0.36 & 0.36 & - & - & & - & - & - & 0.50 & - & - & - & - & - & - \\
\hline & 0.73 & 1.00 & 0.73 & 0.82 & 0.50 & - & 0.68 & 0.68 & - & - & & 0.19 & 0.13 & 0.44 & - & 0.50 & 0.00 & - & - & 0.50 & 0.00 \\
\hline & - & - & - & - & - & 0.50 & - & - & - & - & & 0.69 & 0.63 & 0.94 & - & 1.00 & 0.50 & - & - & 1.00 & 0.50 \\
\hline & 0.55 & 0.82 & 0.55 & 0.64 & 0.32 & - & 0.50 & 0.50 & - & - & & - & - & - & - & - & - & 0.50 & - & - & - \\
\hline & 0.55 & 0.82 & 0.55 & 0.64 & 0.32 & - & 0.50 & 0.50 & - & - & & - & - & - & - & - & - & - & 0.50 & - & - \\
\hline & - & - & - & - & - & - & - & - & 0.50 & - & & 0.19 & 0.13 & 0.44 & - & 0.50 & 0.00 & - & - & 0.50 & 0.00 \\
\hline & - & - & - & - & - & - & - & - & - & 0.50 & & 0.69 & 0.63 & 0.94 & - & 1.00 & 0.50 & - & - & 1.00 & 0.50 \\
\hline \multirow{10}{*}{$P_{3}=$} & 0.50 & 0.60 & 0.20 & - & 0.20 & 0.70 & 0.70 & 0.70 & - & 0.30 & \multirow{10}{*}{$P_{4}=$} & 0.50 & 0.43 & - & - & 0.64 & 0.29 & - & 0.79 & 0.64 & - \\
\hline & 0.40 & 0.50 & 0.10 & - & 0.10 & 0.60 & 0.60 & 0.60 & - & 0.20 & & 0.57 & 0.50 & - & - & 0.71 & 0.36 & - & 0.86 & 0.71 & - \\
\hline & 0.80 & 0.90 & 0.50 & - & 0.50 & 1.00 & 1.00 & 1.00 & - & 0.60 & & - & - & 0.50 & - & - & - & - & - & - & - \\
\hline & - & - & - & 0.50 & - & - & - & - & - & - & & - & - & - & 0.50 & - & - & - & - & - & - \\
\hline & 0.80 & 0.90 & 0.50 & - & 0.50 & 1.00 & 1.00 & 1.00 & - & 0.60 & & 0.36 & 0.29 & - & - & 0.50 & 0.14 & - & 0.64 & 0.50 & - \\
\hline & 0.30 & 0.40 & 0.00 & - & 0.00 & 0.50 & 0.50 & 0.50 & - & 0.10 & & 0.71 & 0.64 & - & - & 0.86 & 0.50 & - & 1.00 & 0.86 & - \\
\hline & 0.30 & 0.40 & 0.00 & - & 0.00 & 0.50 & 0.50 & 0.50 & - & 0.10 & & - & - & - & - & - & - & 0.50 & - & - & - \\
\hline & 0.30 & 0.40 & 0.00 & - & 0.00 & 0.50 & 0.50 & 0.50 & - & 0.10 & & 0.21 & 0.14 & - & - & 0.36 & 0.00 & - & 0.50 & 0.36 & - \\
\hline & - & - & - & - & - & - & - & - & 0.50 & - & & 0.36 & 0.29 & - & - & 0.50 & 0.14 & - & 0.64 & 0.50 & - \\
\hline & 0.70 & 0.80 & 0.40 & - & 0.40 & 0.90 & 0.90 & 0.90 & - & 0.50 & & - & - & - & - & - & - & - & - & - & 0.50 \\
\hline \multirow{10}{*}{$P_{5}=$} & 0.50 & 0.71 & 0.21 & - & 0.29 & 0.64 & - & 0.43 & - & 0.57 & \multirow{10}{*}{$P_{6}=$} & 0.50 & - & - & - & - & - & - & - & - & - \\
\hline & 0.29 & 0.50 & 0.00 & - & 0.07 & 0.43 & - & 0.21 & - & 0.36 & & - & 0.50 & - & 0.00 & 0.13 & 0.38 & - & - & - & 0.00 \\
\hline & 0.79 & 1.00 & 0.50 & - & 0.57 & 0.93 & - & 0.71 & - & 0.86 & & - & - & 0.50 & - & - & - & - & - & - & - \\
\hline & - & - & - & 0.50 & - & - & - & - & - & - & & - & 1.00 & - & 0.50 & 0.63 & 0.88 & - & - & - & 0.50 \\
\hline & 0.71 & 0.93 & 0.43 & - & 0.50 & 0.86 & - & 0.64 & - & 0.79 & & - & 0.88 & - & 0.38 & 0.50 & 0.75 & - & - & - & 0.38 \\
\hline & 0.36 & 0.57 & 0.07 & - & 0.14 & 0.50 & - & 0.29 & - & 0.43 & & - & 0.63 & - & 0.13 & 0.25 & 0.50 & - & - & - & 0.13 \\
\hline & - & - & - & - & - & - & 0.50 & - & - & - & & - & - & - & - & - & - & 0.50 & - & - & - \\
\hline & 0.57 & 0.79 & 0.29 & - & 0.36 & 0.71 & - & 0.50 & - & 0.64 & & - & - & - & - & - & - & - & 0.50 & - & - \\
\hline & - & - & - & - & - & - & - & - & 0.50 & - & & - & - & - & - & - & - & - & - & 0.50 & - \\
\hline & 0.43 & 0.64 & 0.14 & - & 0.21 & 0.57 & - & 0.36 & - & 0.50 & & - & 1.00 & - & 0.50 & 0.63 & 0.88 & - & - & - & 0.50 \\
\hline
\end{tabular}

TABLE III

EXPERTS' OPINIONS COMPLETED WITH PREFERENCES INJECTED FROM TRUSTED EXPERTS - FIRST CASE

\begin{tabular}{|c|c|c|c|c|c|c|c|c|c|c|c|c|c|c|c|c|c|c|c|c|c|}
\hline \multirow{10}{*}{$P_{1}=$} & 0.50 & .77 & 0.50 & 0.59 & 0.27 & 0.32 & 0.45 & 0.45 & 0.62 & 0.35 & \multirow{10}{*}{$P_{2}=$} & 0.50 & 0.44 & 0.75 & 0.38 & 0.81 & 0.31 & 0.70 & 0.69 & 0.81 & 0.31 \\
\hline & 23 & 0.50 & .23 & 0.32 & 0.00 & 0.14 & 0.18 & 0.18 & 0.44 & .16 & & 0.56 & 0.50 & 0.81 & 0.37 & 0.88 & 0.38 & 0.69 & 0.68 & 0.88 & .38 \\
\hline & 0.50 & 0.77 & 0.50 & 0.59 & 0.27 & 0.27 & 0.45 & 0.45 & 0.57 & 0.30 & & 0.25 & 0.19 & 0.50 & 0.26 & 0.56 & 0.06 & 0.58 & 0.57 & 0.56 & .06 \\
\hline & 0.41 & 0.68 & 0.41 & 0.50 & 0.18 & 0.21 & 0.36 & 0.36 & 0.51 & 0.23 & & .42 & 0.46 & 0.54 & 0.50 & 0.62 & 0.37 & 0.65 & 0.64 & 64 & .28 \\
\hline & 0.73 & 1.00 & 0.73 & 0.82 & 0.50 & 0.41 & 0.68 & 0.68 & 0.71 & 0.44 & & 0.19 & 0.13 & 0.44 & 0.21 & 0.50 & 0.00 & 0.53 & 0.52 & 0.50 & 0.00 \\
\hline & 0.61 & 0.79 & 0.66 & 0.63 & 0.52 & 0.50 & 0.53 & 0.60 & 0.73 & 0.46 & & 0.69 & 0.63 & 0.94 & 0.46 & 1.00 & 0.50 & 0.78 & 0.77 & 1.00 & 0.50 \\
\hline & 0.55 & 0.82 & 0.55 & 0.64 & 0.32 & 0.30 & 0.50 & 0.50 & 0.60 & 0.33 & & 0.20 & 0.24 & 0.32 & 0.11 & 0.40 & 0.15 & 0.50 & 0.42 & & .06 \\
\hline & 55 & 0.82 & 0.55 & 0.64 & 0.32 & 0.33 & 0.50 & 0.50 & 0.63 & 0.36 & & 0.25 & 0.29 & 0.37 & 0.16 & 0.45 & 0.19 & 0.48 & 0.50 & 0.47 & 0.11 \\
\hline & 31 & 0.50 & 0.36 & 0.33 & 0.22 & 0.14 & 0.23 & 0.30 & 0.50 & 0.16 & & 0.19 & 0.13 & 0.44 & 0.12 & 0.50 & 0.00 & 0.44 & 0.43 & 0.50 & 0.00 \\
\hline & 59 & 0.77 & .63 & 0.60 & .49 & 0.41 & 0.50 & 0.57 & 0.71 & 0.5 & & 0.69 & 0.63 & 0.94 & 0.55 & 1.00 & 0.50 & 0.87 & 0.86 & 1.00 & 0.50 \\
\hline \multirow{10}{*}{$P_{3}=$} & 0 & 60 & 20 & 32 & 20 & .7 & & & & & \multirow{10}{*}{$P_{4}=$} & & & & & & & & 79 & & .34 \\
\hline & 0.40 & 0.50 & 0.10 & 0.21 & 0.10 & 0.60 & 0.60 & 0.60 & 0.52 & 0.20 & & 0.57 & 0.50 & 0.38 & 0.47 & 0.71 & 0.36 & 0.57 & 0.86 & 0.71 & 0.30 \\
\hline & 0.80 & 0.90 & 0.50 & 0.54 & 0.50 & 1.00 & 1.00 & 1.00 & 0.84 & 0.60 & & 0.58 & 0.62 & 0.50 & 0.59 & 0.62 & 0.53 & 0.69 & 0.79 & 0.69 & 0.42 \\
\hline & 0.48 & 0.62 & 0.26 & 0.50 & 0.30 & 0.67 & 0.65 & 0.65 & 0.64 & 0.32 & & 0.46 & 0.50 & 0.38 & 0.50 & 0.50 & 0.41 & 0.57 & 0.67 & 0.57 & 0.30 \\
\hline & 80 & 0.90 & 0.50 & 0.53 & 0.50 & 1.00 & 1.00 & 1.00 & 0.84 & 0.60 & & 0.36 & 0.29 & 0.38 & 0.47 & 0.50 & 0.14 & 0.57 & .64 & 0.50 & .30 \\
\hline & 30 & 0.40 & 0.00 & 0.16 & 0.00 & 0.50 & 0.50 & 0.50 & 0.47 & 0.10 & & 0.71 & 0.64 & 0.47 & 0.56 & 0.86 & 0.50 & 0.66 & 1.00 & 0.86 & 0.39 \\
\hline & 30 & 0.40 & 00 & 0.11 & 00 & 0.50 & 0.50 & 0.50 & 0.42 & 0.10 & & 0.37 & 0.40 & 0.28 & 37 & 0.40 & 31 & 0.50 & 57 & 47 & .20 \\
\hline & 30 & 0.40 & 0.00 & 0.11 & 0.00 & 0.50 & 0.5 & 0.50 & 0.42 & 0.1 & & 1 & 0. & 0.2 & & 0. & & & 50 & & 0.13 \\
\hline & 0.24 & 0.38 & 0.02 & 0.09 & 0.06 & 0.43 & 0.41 & 0.41 & 0.50 & 0.08 & & 0.36 & 0.29 & 0.24 & 0.33 & 0.50 & 0.14 & 0.43 & 0.64 & 0.50 & 0.16 \\
\hline & 0.70 & 0.80 & 0.40 & 0.52 & 0.40 & 0.90 & 0.90 & 0.90 & 0.83 & 0.50 & & 0.66 & 0.70 & 0.58 & 0.67 & 0.70 & 0.61 & 0.77 & 0.87 & 0.77 & 0.50 \\
\hline \multirow{10}{*}{$P_{5}=$} & 0.50 & 0.71 & 21 & 0.49 & 29 & 0.6 & 0 & & & & \multirow{10}{*}{$P_{6}=$} & & & & & & & & & & .33 \\
\hline & 0.29 & 0.50 & 0.00 & 0.28 & .07 & 0.43 & 0.44 & 0.21 & 0.46 & 0.36 & & 0.29 & 0.50 & 0.29 & 0.00 & 0.13 & .38 & 0.20 & .36 & 0.46 & 0.00 \\
\hline & 0.79 & 1.00 & 0.50 & 0.69 & 0.57 & 0.93 & 0.85 & 0.71 & 0.86 & 0.86 & & 0.43 & 0.61 & 0.50 & 31 & 0.44 & 50 & 34 & 50 & 61 & 0.32 \\
\hline & 0.47 & 0.68 & 0.28 & 0.50 & 0.34 & 0.62 & 0.6 & 0.45 & 0.64 & 0.49 & & 0.45 & 1.00 & 0.46 & 0.50 & 0.63 & & 0.37 & 52 & 0.63 & 0.50 \\
\hline & 0.71 & 0.93 & 0.43 & 0.63 & 0.50 & 0.86 & 0.79 & 0.64 & 0.80 & 0.79 & & 0.46 & 0.88 & 0.46 & 0.38 & 0.50 & 0.75 & 0.37 & 0.52 & 0.63 & 0.38 \\
\hline & 0.36 & 0.57 & 0.07 & 0.35 & 0.14 & 0.50 & 0.51 & 0.29 & 0.52 & 0.43 & & 0.39 & 0.63 & 0.40 & 0.13 & 0.25 & 0.50 & 0.31 & 0.46 & 0.57 & 0.13 \\
\hline & 32 & 0.52 & 12 & 0.31 & .18 & 0.46 & 0.50 & 0.29 & 0.48 & 0.33 & & 0.29 & 0.47 & 0.29 & 0.17 & 0.30 & 0.36 & 0.50 & 0.35 & 0.46 & 0.18 \\
\hline & 57 & 0.79 & 0.29 & 0.48 & 0.36 & 0.71 & 0.64 & 0.50 & 0.66 & 0.64 & & 0.36 & 0.54 & 0.37 & 0.25 & 0.38 & 0.44 & 0.28 & 0.50 & 0.54 & 0.26 \\
\hline & 0.23 & 0.44 & 0.04 & 0.23 & 0.10 & 0.38 & 0.38 & 0.21 & 0.50 & 0.25 & & 0.26 & 0.44 & 0.26 & 0.14 & 0.27 & 0.33 & 0.17 & 0.32 & 0.50 & 0.15 \\
\hline & 0.43 & 0.64 & 0.14 & 0.48 & 0.21 & 0.57 & 0.63 & 0.36 & 0.65 & 0.50 & & 0.57 & 1.00 & 0.58 & 0.50 & 0.63 & 0.88 & 0.48 & 0.64 & 0.75 & 0.50 \\
\hline
\end{tabular}



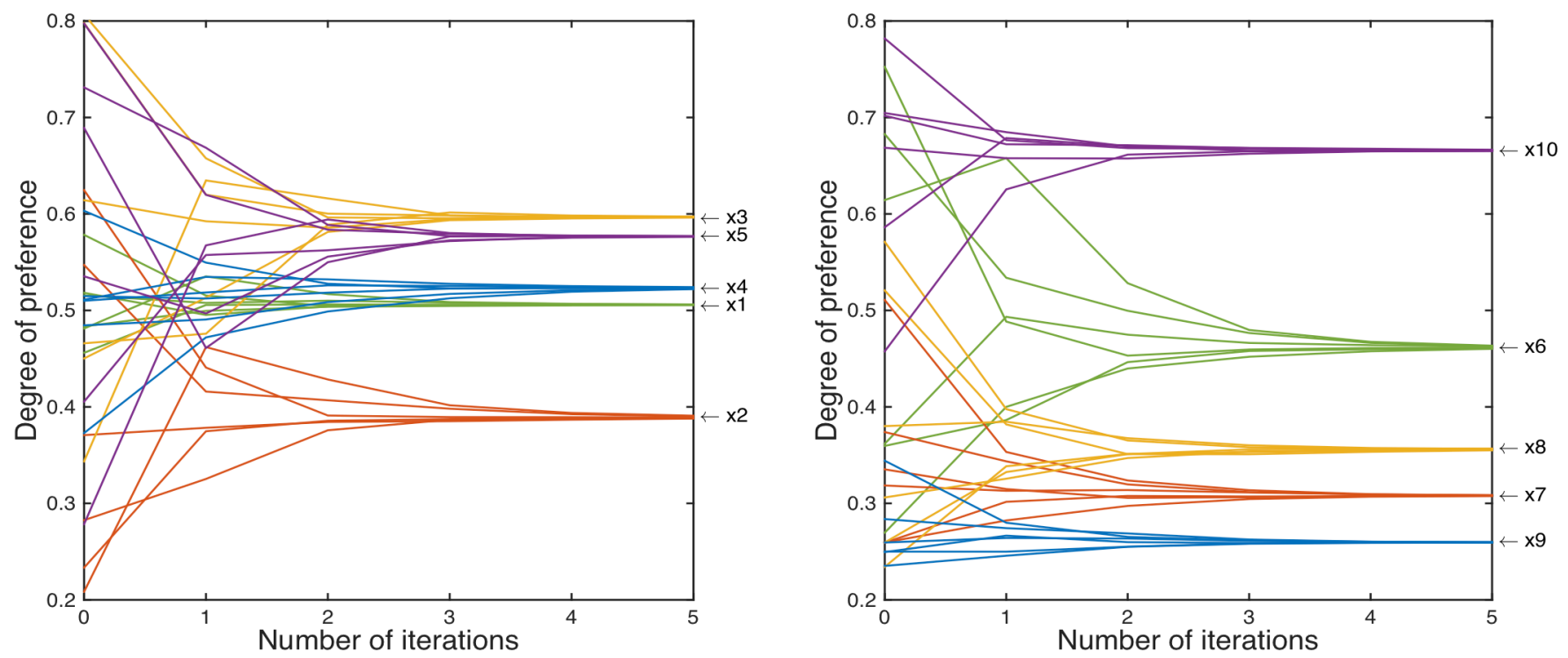

Fig. 5. Evolution of experts' preferences thanks to the influence model - first case. The $x$-axis represents the number of performed iterations, the $y$-axis represents the dominance degree of each alternatives for each expert at a given iteration. Different colors correspond to different alternatives whose identifier is shown on the right. The first 5 alternatives are plotted on the left, the last 5 on the right.

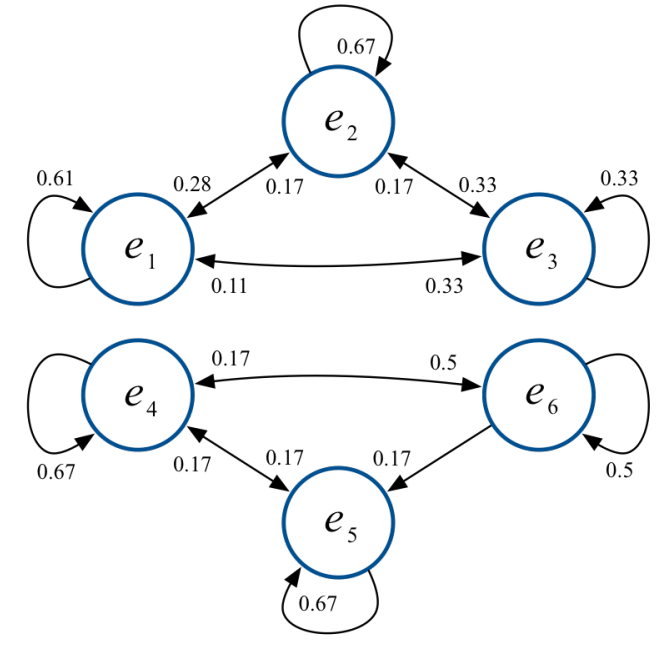

Fig 6. The generated SIN - second case

$$
x_{10} \gg x_{3} \geq x_{5}>x_{4} \geq x_{1}>x_{6} \gg x_{2} \geq x_{8}>x_{7}>x_{9} .
$$

Figure 5 shows the evolution, through the 5 iterations, of the dominance degrees associated to each alternative for the involved experts, which elucidates the convergence process versus the final preferences. The figure also allows to easily perceive the final ranking between alternatives but also observe the process dynamics that led to the generation of the final decision. For example, it can be noticed that the most controversial alternatives have been $x_{6}$ and $x_{2}$ since the convergence on them was reached later than for the other alternatives.

\section{B. Second Case: Lack of Convergence}

A special case for the model is when the matrix $W$ does not respect the conditions for convergence. Let suppose that the six experts of the previous example provide the same opinions on alternatives but different fuzzy rankings of experts (as shown in Table IV). By applying equations (13)-(14), the fuzzy rankings of experts are converted in FPRs and, then, used to build an SIN via equations (16)-(17). The obtained SIN, shown in Figure 6, can be summarized by the following fuzzy adjacency matrix:

$$
W=\left(\begin{array}{cccccc}
0.61 & 0.28 & 0.11 & 0 & 0 & 0 \\
0.17 & 0.67 & 0.17 & 0 & 0 & 0 \\
0.33 & 0.33 & 0.33 & 0 & 0 & 0 \\
0 & 0 & 0 & 0.67 & 0.17 & 0.17 \\
0 & 0 & 0 & 0.17 & 0.67 & 0.17 \\
0 & 0 & 0 & 0.5 & 0 & 0.5
\end{array}\right)
$$

Like in the previous case, the experts are initially in disagreement but, unlike the previous case, they grant their trust only to a small subset of colleagues so as to create two unconnected

TABLE IV

COLLECTED FUZZY RANKINGS OF ALTERNATIVES AND EXPERTS (LEFT) AND COMPLETED FUZZY RANKINGS OF ALTERNATIVES (RIGHT) - SECOND CASE

\begin{tabular}{ccc|c}
\hline \hline Expert & Fuzzy rankings of alternatives & Fuzzy rankings of experts & \multicolumn{1}{c}{ Completed fuzzy rankings of alternatives } \\
\hline$e_{1}$ & $x_{5} \gg x_{7} \approx x_{8} \geq x_{1} \approx x_{3}>x_{4} \gg x_{2}$ & $e_{1}>e_{2} \geq e_{3}$ & $x_{10} \geq x_{5} \gg x_{6}>x_{7} \approx x_{8} \geq x_{1} \approx x_{3} \gg x_{4} \gg x_{9}>x_{2}$ \\
$e_{2}$ & $x_{10} \approx x_{6}>x_{2} \geq x_{1} \gg x_{3} \geq x_{9} \approx x_{5}$ & $e_{2} \gg e_{1} \approx e_{3}$ & $x_{10} \geq x_{6} \gg x_{2} \geq x_{1} \gg x_{7} \approx x_{8} \approx x_{4} \geq x_{3}>x_{5}>x_{9}$ \\
$e_{3}$ & $x_{3} \approx x_{5}>x_{10} \gg x_{1}>x_{2}>x_{6} \approx x_{7} \approx x_{8}$ & $e_{1} \approx e_{2} \approx e_{3}$ & $x_{3} \approx x_{5} \gg x_{10} \gg x_{1} \gg x_{2} \geq x_{4}>x_{6} \approx x_{7} \approx x_{8} \geq x_{9}$ \\
$e_{4}$ & $x_{6}>x_{2} \geq x_{1}>x_{9} \approx x_{5}>x_{8}$ & $e_{4}>e_{5} \approx e_{6}$ & $x_{6} \geq x_{3} \gg x_{4} \approx x_{2} \geq x_{1}>x_{10}>x_{5} \geq x_{7} \approx x_{9} \gg x_{8}$ \\
$e_{5}$ & $x_{3}>x_{5} \gg x_{8}>x_{1}>x_{10}>x_{6}>x_{2}$ & $e_{5} \geq e_{4} \approx e_{6}$ & $x_{3}>x_{5} \gg x_{8} \geq x_{4}>x_{1}>x_{10} \geq x_{9} \approx x_{7} \geq x_{6}>x_{2}$ \\
$e_{6}$ & $x_{10} \approx x_{4}>x_{5} \gg x_{6}>x_{2}$ & $e_{4} \approx e_{6}>e_{5}$ & $x_{4} \approx x_{10} \gg x_{5} \gg x_{1} \geq x_{6} \gg x_{3} \geq x_{7}>x_{9}>x_{2}>x_{8}$ \\
\hline \hline
\end{tabular}



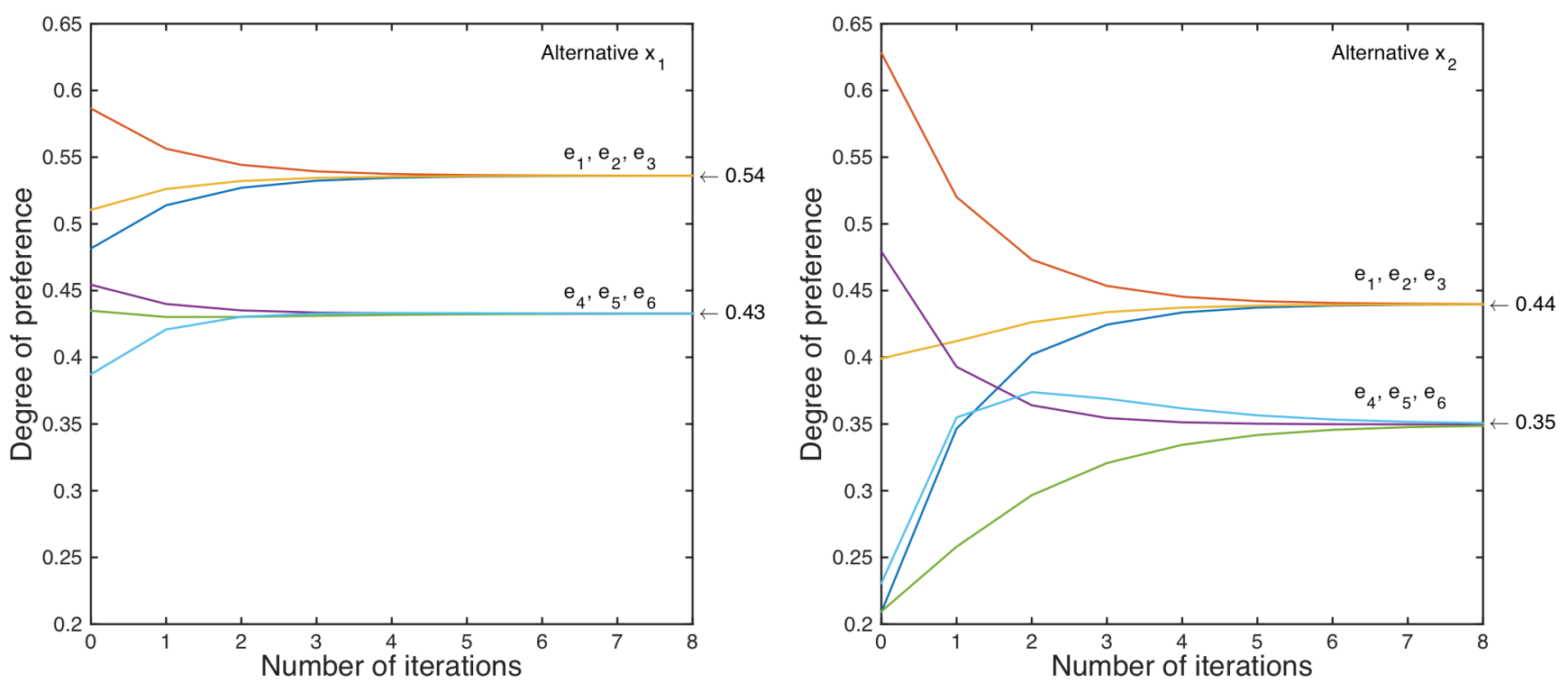

Fig. 7. Evolution of experts' preferences for the alternatives $x_{1}$ (on the left) and $x_{2}$ (on the right) - second case. The $x$-axis represents the number of performed iterations, the $y$-axis represents the dominance degree of the plotted alternative for each expert at a given iteration. Different colors correspond to different experts. Identifiers for experts and alternatives are shown on the right.

subgroups. As it can be seen from the figure (but it is also evident from the matrix $W$ ), experts $e_{1}, e_{2}$ and $e_{3}$ do not provide trust information regarding experts $e_{4}, e_{5}$ and $e_{6}$ so their preferences are not mutually influenced by the model. It is easy to demonstrate that $W$ does not meet the conditions for convergence since it is impossible to find a positive integer $l$ so that every element in at least one column of $W^{l}$ is positive. So it is expected that the influence process does not converge.

Since the fuzzy rankings about alternatives are the same as in the previous example, after conversion, the obtained FPRs are the same already shown in Table II. Obtained FPRs are then completed according to the new SIN and used as input for the influence model (the last column of Table IV reports the completed FPRs converted back into fuzzy rankings). After 8 interactions, each of the two subgroups of experts reaches internal consensus on a single FPR but the FPRs obtained by the two subgroups of experts are different (the two FPRs are reported in Table V).

The evolution of the dominance degree of the first two alternatives is exemplified in Figure 7. Equation (2) is then used to aggregate the FPRs coming from the two subgroups of experts and the resulting dominance degree associated to each alternative is: $\phi\left(x_{1}\right)=0.48, \quad \phi\left(x_{2}\right)=0.39, \quad \phi\left(x_{3}\right)=0.51$, $\phi\left(x_{4}\right)=0.43, \phi\left(x_{5}\right)=0.49, \phi\left(x_{6}\right)=0.54, \phi\left(x_{7}\right)=0.36$, $\phi\left(x_{8}\right)=0.34, \phi\left(x_{9}\right)=0.28, \phi\left(x_{1}\right)=0.6$. Again, the final group solution is $x_{10}$, although the new collective fuzzy ranking of alternatives is:

$$
x_{10} \gg x_{6}>x_{3} \geq x_{5} \approx x_{1}>x_{4}>x_{2}>x_{7} \geq x_{8} \gg x_{9}
$$

\section{Comparative Evaluation}

As confirmed by the preceding experiments, our model offers a greater flexibility with respect to that proposed in [8], based on a predefined SIN. Instead, in our model the SIN is estimated from opinions expressed by the experts in the same form of preferences on alternatives. Moreover, by asking experts to place themselves in the defined rankings, we avoid the complication of defining a numerical level representing the susceptibility of each expert to influence (like in [8]) or a numerical level representing interpersonal trust as in [11] and [12].

In [9], social influence is calculated and represented in form of tie strength between members of a social network by combining the number of their common connections with the number of their direct interactions. Despite this method automates the influence estimation process, it does not provide any guarantee that the strength of a tie on a social network is a good approx-

TABLE V

THE INFLUENCED FPRS OBTAINED WITHIN THE FIRST SUBGROUP (LEFT) AND THE SECOND SUBGROUP (RIGHT) - SECOND CASE

\begin{tabular}{|c|c|c|c|c|c|c|c|c|c|c|c|c|c|c|c|c|c|c|c|c|c|}
\hline \multirow{10}{*}{$P^{\prime}=$} & 0,50 & 0,58 & ,56 & 0,58 & 0,51 & 0,41 & 0,58 & 0,58 & 0,72 & 0,29 & \multirow{10}{*}{$P^{\prime \prime}=$} & 0,50 & 0,52 & 0,25 & 0,29 & 0,49 & 0,41 & 0,32 & 0,63 & 0,55 & 0,44 \\
\hline & 0,42 & 0,50 & 0,48 & 0,45 & 0,43 & 0,36 & 0,46 & 0,46 & 0,67 & & & ,43 & 0,50 & 0,15 & 0,15 & 0,41 & 0,38 & 0,23 & 0,60 & 0,52 & 0,29 \\
\hline & 0,44 & 0,52 & 0,50 & 0,55 & 0,45 & 0,33 & 0,57 & 0,57 & 0,63 & 0,2 & & ,56 & 0,69 & 0,50 & 0,3 & 0,56 & 59 & 41 & 0,66 & 55 & 56 \\
\hline & 0,36 & 0,48 & 0,39 & 0,50 & 0,31 & 0,28 & 0,44 & 0,44 & 0,52 & 0 , & & & & 30 & & & 0,60 & & 0,58 & & 51 \\
\hline &, 49 & 0,57 & 0,55 & 0,63 & 0,50 & 0,35 & 0,65 & 0,65 & 0,65 & 0,23 & & 0,46 & 0,59 & 0,29 & 0,32 & 0,50 & 0,47 & 0,33 & 0,63 & 0,52 & ,49 \\
\hline & 0,58 & 0,63 & 0,66 & 0,59 & 0,63 & 0,50 & 0,63 & 0,63 & 0,80 & 0,37 & & 0,54 & 0,62 & 0,25 & 0,25 & 0,53 & 0,50 & 0,33 & 0,71 & 0,64 &, 40 \\
\hline & 0,40 & 0,53 & 0,42 & 0,48 & 0,34 & 0,32 & 0,50 & 0,48 & 0,54 & 0,16 & & 0,30 & 0,43 & 0,14 & 0,17 & 0,32 & 0,33 & 0,50 & 0,41 & 0,34 & 0,30 \\
\hline & 0,40 & 0,53 & 0,42 & 0,48 & 0,34 & 0,32 & 0,48 & 0,50 & 0,54 & 0,16 & & 0,30 & 0,35 & 0,15 & 0,17 & 0,32 & 0,24 & 0,20 & 0,50 & 0,37 & 0,33 \\
\hline & 0,23 & 0,28 & 0,32 & 0,28 & 0,30 & 0,13 & 0,32 & 0,32 & 0,50 & 0,02 & & 0,33 & 0,39 & 0,16 & 0,19 & 0,39 & 0,27 & 0,23 & 0,51 & 0,50 & 0,33 \\
\hline & 0,70 & 0,75 & 0,78 & 0,73 & 0,76 & 0,61 & 0,79 & 0,79 & 0,91 & 0,50 & & 0,42 & 0,66 & 0,24 & 0,32 & 0,46 & 0,55 & 0,31 & 0,52 & 0,45 & 0,50 \\
\hline
\end{tabular}


TABLE VI

COMPARISON WITH OTHER MODELS

\begin{tabular}{l|llll}
\hline \hline & \multicolumn{1}{|c}{ Our model } & Model proposed in [8] & \multicolumn{1}{c}{ Model proposed in [9] } & Models in [11] and [12] \\
\hline $\begin{array}{l}\text { Estimation } \\
\text { of Social Influence }\end{array}$ & $\begin{array}{l}\text { Based on fuzzy rankings } \\
\text { of experts }\end{array}$ & Based on a predefined SIN & $\begin{array}{l}\text { Based on social network } \\
\text { analysis }\end{array}$ & $\begin{array}{l}\text { Based on numerical } \\
\text { trust statements }\end{array}$ \\
of Social Influence & SIN & SIN & Normalized tie strength & Normalized trust level \\
& $\begin{array}{l}\text { Estimation of Missing } \\
\text { Preferences }\end{array}$ & & $\begin{array}{l}\text { Estimation of Experts' } \\
\text { Importance }\end{array}$ & $\begin{array}{l}\text { Estimation of Experts' } \\
\text { Importance }\end{array}$ \\
$\begin{array}{l}\text { Applications } \\
\text { of Social Influence }\end{array}$ & $\begin{array}{l}\text { Evolution of Preferences } \\
\text { Selection of the Best } \\
\text { Alternative }\end{array}$ & Evolution of Preferences & $\begin{array}{l}\text { Estimation of Missing } \\
\text { Preferences }\end{array}$ & \\
\hline \hline
\end{tabular}

imation of how an opinion can be influenced with respect to a specific decision problem. Moreover it requires that all experts are active members of the same social network.

With respect to the applications of social influence, it should be noted that in our model the influence in not used to estimate a global importance level for each expert (like in [9], [11] and [12]) but to let the preference of each expert gradually evolve simulating the effect of social interaction (like in [8]). In addition (like in [9]) we also use influence as a mean to estimate missing preferences in case of incomplete information. This is possible because, in our model, influence evolution can be operated directly on experts' FPRs rather than on utility vectors extracted from FPRs (like in [8]).

Simulating the natural evolution of opinions thanks to discussion, our model also tries to obtain the convergence between the experts' opinions. This is a distinctive feature with respect to existing models because social influence also impacts the preferences aggregation phase. In such sense, our model can be also used to support automated consensus processes. Table VI summarizes the differences and the advantages of the proposed model with respect to other existing ones.

\section{CONCLUSIONS}

Despite its prominent role in opinion formation, social influence seems to be almost disregarded by current GDM models. Aiming at filling this gap, we have developed in this paper a new GDM model able to take into account social influence between experts during the process and to estimate how experts' opinion change according to its effects. In this model the concept of social influence is strictly interconnected with that of interpersonal trust according to the intuition that the more an expert trusts in the capability of another expert, the more his opinion is influenced by the trusted expert, especially in presence of incomplete information i.e. when experts are unable to express an opinion on any of the alternatives.

Fuzzy rankings are used to represent experts' opinions regarding both their preferences on the set of alternatives and their trust on other experts. This opinion representation format is user friendly and less vulnerable to inconstancy than commonly used FPRs. Fuzzy rankings of experts are used to build an SIN that specifies the structure and the level of experts' interpersonal influence. The network is used, in turn, to estimate missing preferences and to let them evolve simulating the effects of experts' interaction before aggregating them for the selection of the best alternative. The time complexity of the whole process is polynomial and limited by $\mathcal{O}\left(m \cdot n^{3} \log n\right)$ where $m$ is the number of experts and $n$ the number of alternatives. It has been also demonstrated that under certain conditions, experts' opinion naturally converge to a final collective opinion.

We believe that the defined model leads to a more accurate representation of the GDM process by formalizing important aspects that are usually disregarded by other models. On the other hand, we estimate the level of social influence based on interpersonal trust without considering other psychological traits like leadership, charisma, persuasive ability, etc. that could strengthen or weaken influence when real interactions between experts take place. Nevertheless, we believe that the exclusion of these additional traits is advantageous and enables to reach more objective decisions.

\section{APPENDIX}

To improve readability, some demonstrations have been removed from the main text and presented here. In particular we prove below two statements made in sections III.A and III.C.

Proposition 1 (additive consistency of FPRs generated from fuzzy rankings). If $P$ is a $n \times n$ FPR generated from a fuzzy ranking $R$ according to equations (13) and (14), then the elements of $P$ that exist verify the additive consistency property.

Proof. A $n \times n$ FPR $P=\left(p_{i j}\right)$ is additive consistent if $p_{i j}+p_{j k}+p_{k i}=1.5 \forall i, j, k \in\{1, \ldots, n\}$. Based on equation (14) we can write:

$$
\begin{aligned}
& p_{i j}+p_{j k}+p_{k i}=\frac{1}{2}\left(1+\frac{r\left(x_{j}\right)-r\left(x_{i}\right)}{r \max -1}\right)+ \\
& +\frac{1}{2}\left(1+\frac{r\left(x_{k}\right)-r\left(x_{j}\right)}{r \max -1}\right)+\frac{1}{2}\left(1+\frac{r\left(x_{i}\right)-r\left(x_{k}\right)}{r \max -1}\right)= \\
& =\frac{3}{2}+\frac{r\left(x_{j}\right)-r\left(x_{i}\right)+r\left(x_{k}\right)-r\left(x_{j}\right)+r\left(x_{i}\right)-r\left(x_{k}\right)}{2 r \max -2} .
\end{aligned}
$$

For $r \max \neq 1$ and because its fraction numerator is 0 , we have that $p_{i j}+p_{j k}+p_{k i}=3 / 2+0=1.5$ proofing that $P$ is additive consistent. The case $\operatorname{rmax}=1$, which leads to a $0 / 0$ indeterminate form, is treated separately in section III.A by setting $p_{i j}=0.5 \forall i, j \in\{1, \ldots, n\}$. In this case the proof that $P$ is additive consistent is trivial given that: $p_{i j}+p_{j k}+p_{k i}=$ 
$0.5+0.5+0.5=1.5 \forall i, j, k \in\{1, \ldots, n\}$.

Proposition 2 (convergence of the influence model). If $Q=(0,1)$ in equation $(22)$ and there exists a positive integer $l$ so that every element in at least one column of the SIN fuzzy adjacency matrix $W^{l}$ is positive, then all the FPRs $P_{k}^{(t)}$ for $k \in\{1, \ldots, m\}$ converge to the same FPR.

Proof. Combining equation (21) with the definition of the IIOWA operator provided by equations (8) and (9), we obtain that, being $p_{k}^{(t)}$ a generic element belonging to the FPR $P_{k}^{(t)}$ for $k \in\{1, \ldots, m\}$ and $t>1$ :

$p_{k}^{(t)}=I-I O W A_{Q}\left(\left(p_{1}^{(t-1)}, w_{k 1}\right), \ldots,\left(p_{m}^{(t-1)}, w_{k m}\right)\right)=$

$=\sum_{i=1}^{m}\left(\mu_{Q}\left(\frac{S(i)}{S(m)}\right)-\mu_{Q}\left(\frac{S(i-1)}{S(m)}\right)\right) p_{\sigma(i)}^{(t-1)}$.

where $S(i)=\sum_{j=1}^{i} w_{k \sigma(j)}$ and $\sigma:\{1, \ldots m\} \rightarrow\{1, \ldots m\}$ is a permutation function so that $w_{\sigma(i)} \geq w_{\sigma(i+1)}$ for $i \in$ $\{1, \ldots, m\}$. Being $Q=(0,1)$, by substituting $a=0$ and $b=1$ in equation (3) we obtain $\mu_{Q}(y)=\frac{y-0}{1-0}=y$ for $0 \leq y \leq 1$. Given that $S(i)$ and $S(m)$ are positive number so that $S(m) \geq S(i)$ for $1 \leq i \leq m$, then we can say that $0 \leq \frac{S(i)}{S(m)} \leq$ 1 so $\mu_{Q}\left(\frac{S(i)}{S(m)}\right)=\frac{S(i)}{S(m)}$. By substituting this in the preceding equation we obtain:

$p_{k}^{(t)}=\sum_{i=1}^{m}\left(\frac{S(i)}{S(m)}-\frac{S(i-1)}{S(m)}\right) p_{\sigma(i)}^{(t-1)}=$

$=\sum_{i=1}^{m} \frac{\sum_{j=1}^{i} w_{k \sigma(j)}-\sum_{j=1}^{i-1} w_{k \sigma(j)}}{\sum_{j=1}^{m} w_{k \sigma(j)}} p_{\sigma(i)}^{(t-1)}=\sum_{i=1}^{m} \frac{w_{k \sigma(i)}}{\sum_{j=1}^{m} w_{k \sigma(j)}} p_{\sigma(i)}^{(t-1)}$.

Given that $W$ is the fuzzy adjacency matrix of a SIN, thanks to equation (16) we have that $\sum_{j=1}^{m} w_{k j}=1$ for any $k \in$ $\{1, \ldots, m\}$. Being $\sigma$ a permutation function, $\sum_{j=1}^{m} w_{k \sigma(j)}$ simply sum the same elements in a different order so we can say that $\sum_{j=1}^{m} w_{k \sigma(j)}=1$ too. By substituting this in the preceding equation we obtain:

$p_{k}^{(t)}=\sum_{i=1}^{m} w_{k \sigma(i)} p_{\sigma(i)}^{(t-1)}=\sum_{i=1}^{m} w_{k i} p_{i}^{(t-1)}$.

If we build the vector $p^{(t)}=\left(p_{1}^{(t)}, \ldots, p_{m}^{(t)}\right)^{T}$ including the same preference as expressed by all the $m$ experts we can generalize the preceding equation using matrix notation as $p^{(t)}=W p^{(t-1)}=W^{t-1} p^{(1)}$. As explained in [6], $W$ can be regarded as the one-step transition probability matrix of a Markov chain with $m$ states and stationary transition probabilities.

If there exists a positive integer $l$ so that every element in at least one column of $W^{l}$ is positive then the Markov chain is said regular and, thanks to the limit theorem for regular finite Markov chains [46], it exists a value $p$ so that $\lim _{t \rightarrow \infty} p_{k}^{(t)}=$ $p \forall k \in\{1, \ldots, m\}$ i.e. the preferences expressed by the $m$ ex- perts converge to the same value $p$. By extending this result (that regards a generic preference belonging to a FPR) to the whole FPR, we can say that, if conditions are met, all the FPRs $P_{k}^{(t)}$ for $k \in\{1, \ldots, m\}$ converge to the same FPR.

\section{REFERENCES}

[1] Filip FG, Zamfirescu BC, Ciurea C (2017). Computer-Supported Collaborative Decision-Making, Springer

[2] Pedrycz W, Ekel P, Parreiras R (2011). Fuzzy Multicriteria DecisionMaking: Models, Methods and Applications. John Wiley \& Sons.

[3] Cabrerizo FJ, Chiclana F, Al-Hmouz R, Morfeq A, Balamash AS, Herrera-Viedma E (2015). Fuzzy decision making and consensus. Journal of Intelligent and Fuzzy Systems, 29(3):1109-1118.

[4] Zhu B, Xu Z, Zhang R, Hong M (2015). Generalized analytic network process. European Journal of Operational Research, 244(1):277-288.

[5] Zhu B, Xu Z, Xu J (2014). Deriving a ranking from hesitant fuzzy preference relations under group decision making. IEEE transactions on cybernetics, 44(8):1328-1337.

[6] De Groot MH (1974). Reaching a consensus. Journal of American Statistical Association, 69:118-121.

[7] Friedkin N, Johnsen E (1999). Social Influence Networks and Opinion Change. Advances in Group Processes, 16(1):1-29.

[8] Pérez LG, Mata F, Chiclana F, Kou G, Herrera-Viedma E (2016). Modelling influence in group decision making. Soft Computing, 20(4):16531665.

[9] Liang Q, Liao X, Liu J (2017). A social ties-based approach for group decision-making problems with incomplete additive preference relations, Knowledge-Based Systems, 119:68-86.

[10] Artz D, Gil Y (2007). A survey of trust in computer science and the Semantic Web. Journal of Web Semantics: Science, Services and Agents on the World Wide Web, 5(2):58-71.

[11] Wu J, Chiclana F (2014). A social network analysis trust-consensus based approach to group decision-making problems with interval-valued fuzzy reciprocal preference relations. Knowledge-Based Systems 59:97107

[12] Wu J, Chiclana F, Herrera-Viedma E (2015). Trust Based Consensus Model for Social Network in an Incomplete Linguistic Information Context. Applied Soft Computing, 35: 827-839.

[13] Seo F, Sakawa M (1985). Fuzzy multiattribute utility analysis for collective choice. IEEE Transactions on Systems, Man and Cybernetics, $15(1): 45-53$.

[14] Tanino T (1990). On group decision making under fuzzy preferences. In Multiperson decision making using fuzzy sets and possibility theory. Kluwer Academic Publisher, 172-185.

[15] Li RJ (1999). Fuzzy method in group decision making. Computers \& Mathematics with Applications, 38(1):91-101.

[16] Kitainik L (1993). Fuzzy decision procedures with binary relations: towards a unified theory. Springer.

[17] Bezdek JC, Spillman B, Spillman R (1978). A fuzzy relation space for group decision theory. Fuzzy Sets and Systems, 1(4):255-268.

[18] Zhou W, Xu Z, Chen M (2015). Preference relations based on hesitantintuitionistic fuzzy information and their application in group decision making. Computers \& Industrial Engineering, 87(C):163-175.

[19] Pedrycz W, Ekel P, Parreiras R (2011). Construction of Fuzzy Preference Relations. In Fuzzy Multicriteria Decision-Making: Models, Methods and Applications, John Wiley \& Sons, 6:155-191.

[20] Ureña MR, Chiclana F, Morente-Molinera JA, Herrera-Viedma E (2015). Managing Incomplete Preference Relations in Decision Making: A Review and Future Trends. Information Sciences 302(1):14-32.

[21] Wang YM, Fan ZP (2007). Fuzzy preference relations: Aggregation and weight determination. Computers \& Industrial Engineering, 53(1):163172.

[22] Ma J, Fan ZP, Jiang YP, Mao JY, Ma L (2006). A method for repairing the inconsistency of fuzzy preference relations. Fuzzy Sets and Systems, 157(1):20-33.

[23] Yager RR (1993). Families of OWA operators. Fuzzy Sets and Systems, 59(2):125-148.

[24] Malczewski J (2006). Ordered weighted averaging with fuzzy quantifiers: gis-based multicriteria evaluation for land-use suitability analysis. International Journal of Applied Earth Observation \& Geoinformation, 8: $270-277$. 
[25] Peláez JI, Doña JM, Gómez-Ruiz JA (2007). Analysis of OWA operators in decision making for modelling the majority concept. Applied Mathematics and Computation, 186(2):1263-1275.

[26] Chiclana F, Herrera F, Herrera-Viedma E. (1998). Integrating three representation models in fuzzy multipurpose decision making based on fuzzy preference relations. Fuzzy Sets and Systems, 97(1):33-48.

[27] Zadeh LA (1983). A computational approach to fuzzy quantifiers in natural languages. Computers \& Mathematics with Applications, 9(1):149184.

[28] Díaz-Hermida F, Bugarín A (2003). Definition and classification of semi-fuzzy quantifiers for the evaluation of fuzzy quantified sentences. International Journal of Approximate Reasoning. 34(1): 49-88.

[29] Peláez JI, Doña JM, Gómez-Ruiz JA (2007). Analysis of OWA operators in decision making for modelling the majority concept. Applied Mathematics and Computation, 186(2):1263-1275.

[30] Herrera-Viedma E, Chiclana F, Herrera F, Alonso S (2007). Group Decision-Making Model with Incomplete Fuzzy Preference Relations Based on Additive Consistency. IEEE Transactions on Systems, Man, and Cybernetics, Part B: Cybernetics, 37(1):176-189.

[31] Alonso S, Herrera-Viedma E, Chiclana F, Herrera F (2010). A Web Based Consensus Support System for Group Decision Making Problems and Incomplete Preferences. Information Sciences, 180(23):4477-4495.

[32] Alonso S, Chiclana F, Herrera F, Herrera-Viedma E, Alcalá-Fdez J, Porcel C (2008). A consistency-based procedure to estimate missing pairwise preference values, International Journal on Intelligent Systems, 23(2):155-175.

[33] Xu ZS (2004). Goal programming models for obtaining the priority vector of incomplete fuzzy preference relation, International Journal of Approximate Reasoning, 36(3):261-270.

[34] Zhou W, Xu ZS (2016). Asymmetric fuzzy preference relations based on the generalized sigmoid scale and their application in decision making involving risk appetites. IEEE Transactions on Fuzzy Systems, 24(3): 741-756.

[35] Sarabando P, Dias LC, Vetschera R (2016). Group decision making with incomplete information: a dominance and quasi-optimality volumebased approach using Monte-Carlo simulation. International Transactions in Operational Research, In Press, doi: 10.1111/itor.12315.

[36] Alonso S, Herrera-Viedma E, Chiclana F, Herrera F (2009). Individual and Social Strategies to Deal with Ignorance Situations in Multi-Person Decision Making. International Journal of Information Technology and Decision Making, 8(2):313-333.

[37] Yager RR, Filev D (1999). Induced ordered weighted averaging operators. IEEE Transactions on Systems, Man and Cybernetics, 29:141-150.

[38] Chiclana F, Herrera-Viedma E, Herrera F, Alonso S (2007). Some induced ordered weighted averaging operators and their use for solving group decision-making problems based on fuzzy preference relations. European Journal of Operational Research, 182(1):383-399.

[39] Pérez IJ, Cabrerizo FJ, Alonso S, Herrera-Viedma E (2014). A New Consensus Model for Group Decision Making Problems with Non Homogeneous Experts. IEEE Transactions on Systems, Man, and Cybernetics: Systems, 44(4):494-498.

[40] Herrera-Viedma E, Herrera F, Chiclana F, Luque M (2004). Some issues on consistency of fuzzy preference relations. European Journal of Operational Research, 154(1):98-109

[41] Grady CA, He X, Peeta S (2015). Integrating social network analysis with analytic network process for international development project selection, Expert Systems with Applications 42:5128-5138.

[42] Duong TH, Nguyen NT, Truong HB, Nguyen VH (2015). A collaborative algorithm for semantic video annotation using a consensus-based social network analysis, Expert Systems with Applications, 42:246-258.

[43] Capuano N, Loia V, Orciuoli F (2016). A Fuzzy Group Decision Making Model for Ordinal Peer Assessment. IEEE Transactions on Learning Technology, 10(2):247-259.

[44] Bordogna G, Fedrizzi M, Passi G (1997). A Linguistic Modelling of Consensus in Group Decision Making Based on OWA Operators. IEEE Transactions on Systems, Man and Cybernetics, 27:126-132.

[45] Albano G, Capuano N, Pierri A (2017). Adaptive Peer Grading and Formative Assessment. Journal of e-Learning and Knowledge Society, 13(1):147-161.

[46] Doob JL (1990). Stochastic Processes. John Wiley \& Sons.

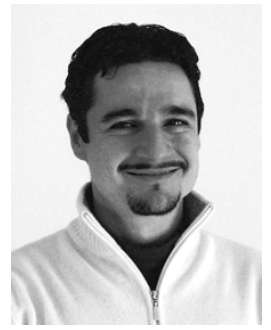

Nicola Capuano received the M.Sc. degree in Computer Science from the University of Salerno, Italy in 1998 . He was Research Assistant at the same University and now he serves as Scientific Officer. His research interests include Computational Intelligence, Fuzzy Systems, Intelligent Tutoring Systems and Knowledge Representation. He is author of about 100 papers.

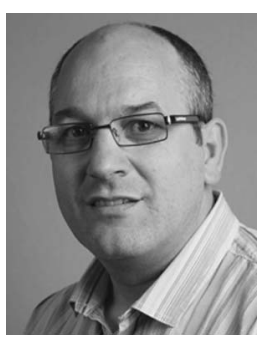

Francisco Chiclana is a Professor of computational intelligence and decision making with the School of Computer Science and Informatics, Faculty of Technology, De Montfort University. He is an Associate Editor and a Guest Editor for several ISI indexed journals. He has organized and chaired special sessions/workshops in many major international conferences in research areas as fuzzy preference modeling, decision support sytems, consensus, recommender systems, social networks, rationality/consistency, aggregation.

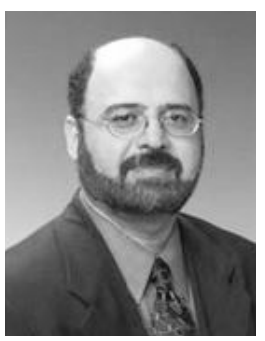

Hamido Fujita is chair professor at Iwate Prefectural University (IPU), Iwate, Japan, as a director of Intelligent Software Systems. $\mathrm{He}$ is the Editor-in-Chief of Knowledge-Based Systems, Elsevier. He is Adjunct professor to Stockholm University, Sweden. He has four international Patents in Software System and Several research projects with Japanese industry and partners. He is vice president of International Society of Applied Intelligence.

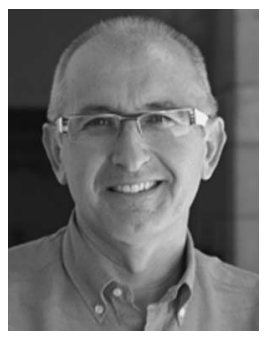

Enrique Herrera-Viedma is currently the Vice-Chancellor of Research and Transfer Knowledge with University of Granada and a Professor of Computer Science. He was identified as a Highly Cited Researcher by Thomson Reuters in Computer Science and Engineering (2014, 2015 and 2016). His current research interests include consensus, linguistic modeling, aggregation, IR, bibliometric, digital libraries, and recommender systems. He serves as Associate Editor in many ISI journals.

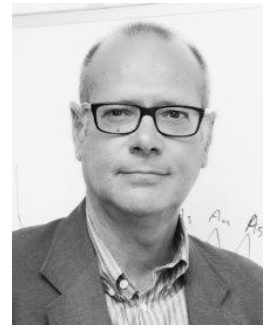

Vincenzo Loia is a Professor of Computer Science at University of Salerno. He is the coeditor-in-chief of Soft Computing and the editor-in-chief of Ambient Intelligence and Humanized Computing, both from Springer. He is an Associate Editor of various ISI journals. He is the author of more than 300 research papers in international journals, books and conference proceedings. His research interests include soft computing, agent technology for technologically complex environments Web intelligence, Situational Awareness. 